\title{
Anomalous temperature dependence of resistivity in quasi-one-dimensional conductors in a strong magnetic field
}

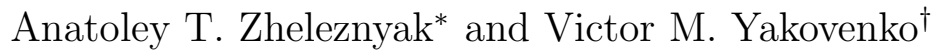 \\ Department of Physics and Center for Superconductivity Research, University of Maryland, \\ College Park, MD 20742-4111 \\ (v.3: 26 Mar 1999 (v.1: 16 Feb 1998), cond-mat/9802172, Eur. Phys. J. B 11, 385-399 (1999))
}

\begin{abstract}
We present a heuristic, semiphenomenological model of the anomalous temperature $(\mathrm{T})$ dependence of resistivity $\rho_{x x}$ recently observed experimentally in the quasi-one-dimensional (Q1D) organic conductors of the (TMTSF) $)_{2} \mathrm{X}$ family in moderately strong magnetic fields. We suggest that a Q1D conductor behaves like an insulator $\left(d \rho_{x x} / d T<0\right)$, when its effective dimensionality is one, and like a metal $\left(d \rho_{x x} / d T>0\right)$, when its effective dimensionality is greater than one. Applying a magnetic field reduces the effective dimensionality of the system and switches the temperature dependence of resistivity between the insulating and metallic laws depending on the magnitude and orientation of the magnetic field. We critically analyze whether various microscopic models suggested in literature can produce such a behavior and find that none of the models is fully satisfactory. In particular, we perform detailed analytical and numerical calculations within the scenario of magneticfield-induced spin-density-wave precursor effect suggested by Gor'kov and find that the theoretical results do not agree with the experimental observations. 72.10.-d, 72.15.Gd, 75.30.Fv, 74.70.Kn
\end{abstract}




\section{EXPERIMENTAL INTRODUCTION}

In recent experiments [1 1 , a very unusual temperature $(T)$ dependence of resistivity was observed in quasi-one-dimensional (Q1D) organic conductors (TMTSF) ${ }_{2} \mathrm{ClO}_{4}$ (at the ambient pressure) and (TMTSF $)_{2} \mathrm{PF}_{6}$ (at a pressure about $9 \mathrm{kbar}$ ) [5] in moderately strong magnetic fields $H$ of the order of $10 \mathrm{~T}$ at $T \lesssim 50 \mathrm{~K}$. Unexpectedly large magnetoresistance in these materials has already attracted attention in earlier measurements 6 [8].

The (TMTSF) ${ }_{2} \mathrm{X}$ materials consist of one-dimensional (1D) conducting chains parallel to the crystal axis a (for general reviews of the (TMTSF) $)_{2} \mathrm{X}$ materials, see Refs. [1,9-12]). The chains are weakly coupled in the two others directions $\mathbf{b}$ and $\mathbf{c}$, the coupling in the $\mathbf{c}$ direction being much weaker than in the $\mathbf{b}$ direction. In zero magnetic field or in the field parallel to the $\mathbf{b}$ axis, the resistivity of (TMTSF $)_{2} \mathrm{PF}_{6}$ along the chains, $\rho_{a a}$, depends on temperature approximately quadratically: $\rho_{a a} \sim T^{2}$ [1,4,13], which is consistent with the standard Fermi-liquid theory [14], provided resistivity is dominated by electron-electron scattering. When a magnetic field is applied along the $\mathbf{c}$ axis, $\rho_{a a}$ does not change appreciably at high temperatures; however, below a certain magnetic-field-dependent temperature $T_{\min } \sim 20 \mathrm{~K}$, resistivity starts to grow with decreasing temperature: $d \rho_{a a} / d T<0$ at $T<T_{\min }$ [1, 3 , [4]. In other words, the behavior of the system changes from metallic, $d \rho_{a a} / d T>0$, to insulating, $d \rho_{a a} / d T<0$, when the temperature is lowered below $T_{\min }$. The temperature $T_{\min }$ increases with the increase of magnetic field. Such a behavior is very surprising in view of the fact that no thermodynamic phase transition is observed in the system at this relatively high temperature $T_{\min } \sim 20 \mathrm{~K}$. (A phase transition into the magnetic-field-induced spindensity-wave (FISDW) state takes place at the transition temperature $T_{c} \sim 2 \mathrm{~K}$, which is

an order of magnitude lower than $T_{\min }$.) As the temperature is lowered further below $T_{\min }$, $\rho_{a a}(T)$ continues to grow until another temperature scale $T_{\max } \sim 8 \mathrm{~K}<T_{\min }$ is reached. Magnetoresistance is huge at $T \sim T_{\max }: \rho_{a a}$ at $H=7.8 \mathrm{~T}$ is about 10 times greater than $\rho_{a a}$ at $H=0$ [4]. At the lower temperatures $T<T_{\max }$, behavior of the system starts to depend crucially on the exact orientation of the magnetic field [4, 15. If the magnetic field 
lies in a plane formed by the direction a of the chains and another integer crystallographic direction, such as $\mathbf{c}$ or $\mathbf{c}+\mathbf{b}, \rho_{a a}$ recovers the metallic behavior $d \rho_{a a} / d T>0$ at $T<T_{\max }$. For other, generic orientations of the magnetic field, $\rho_{a a}$ retains the nonmetallic behavior, either continuing to grow with decreasing temperature: $d \rho_{a a} / d T<0$, or saturating at a high constant value. The temperature $T_{\max }$ does not depend appreciably on the magnetic field. If at $T<T_{\max }$ the magnetic field is rotated in the plane perpendicular to the direction a of the chains, $\rho_{a a}$ exhibits sharp minima when the field is aligned with the integer planes described above (the so-called "magic angles" effect). This effect was discovered experimentally earlier [16 18] following the theoretical suggestion by Lebed' [19,20]. Finally, at low temperatures, the system may enter the magnetic-field-induced spin-density-wave (FISDW) phase at the transition temperature $T_{c} \sim 1-2 \mathrm{~K}$, where $\rho_{a a}$ increases sharply. Other diagonal components of the resistivity tensor of (TMTSF) $)_{2} \mathrm{PF}_{6}$ seem to behave similarly to $\rho_{a a}$ : The angular dependence of $\rho_{c c}$ [4,21] at $T<T_{\max }$ and the temperature dependences of $\rho_{c c}$ [22,23] and $\rho_{b b}$ [23 25] appear to be qualitatively similar to those of $\rho_{a a}$. However, in the very recent measurements [24] it was found that, when the magnetic field is oriented along the magic direction $\mathbf{c}, \rho_{c c}$ monotoniuosly decreases with temperature, whereas $\rho_{a a}$ and $\rho_{b b}$ exhibit a minimum at $T=T_{\min }$. When the magnetic field is tilted away from $\mathbf{c}, \rho_{c c}$ develops a minimum at a temperature much lower than $T_{\min }$ for $\rho_{a a}$ and $\rho_{b b}$.

In (TMTSF $)_{2} \mathrm{ClO}_{4}$, a magnetic field also causes $\rho_{a a}(T)$, as well as the NMR relaxation rate $1 / T_{1}$, to grow with decreasing temperature at $T<T_{\min }$ [2,26], which is an indication of a nonmetallic behavior induced by the magnetic field. On the other hand, it was shown in Refs. 21,27,28] that the angular and temperature dependences of $\rho_{c c}$ in (TMTSF) ${ }_{2} \mathrm{ClO}_{4}$ are quite different from those in (TMTSF $)_{2} \mathrm{PF}_{6}$ and can be interpreted within the standard Fermi-liquid picture. The behavior of (TMTSF) ${ }_{2} \mathrm{ClO}_{4}$ may or may not [28] be complicated by doubling of the crystal period in the $\mathbf{b}$ direction occurring in this material at $T=24 \mathrm{~K}$. To avoid complications, we will focus our theoretical study on (TMTSF) ${ }_{2} \mathrm{PF}_{6}$, which has a simple crystal structure.

The behavior of resistivity in (TMTSF $)_{2} \mathrm{PF}_{6}$ completely contradicts the conventional 
Fermi-liquid picture of a metal with an open Fermi surface. In this picture, applying a magnetic field perpendicular to the direction of the chains should produce no or very little magnetoresistance, should not alter the metallic temperature dependence of resistance, and should exhibit no magic angles effect [29]. Thus, unconventional theoretical approaches are required.

\section{HEURISTIC THEORETICAL PICTURE}

We suggest that the following theoretical picture may qualitatively account for the unusual behavior of (TMTSF $)_{2} \mathrm{PF}_{6}$ [30.

It is well known theoretically (see, for example, Ref. [31]) that the orbital effect of a c-axis magnetic field $H$ on a Q1D conductor is characterized by the cyclotron energy $E_{H}=e b H v_{F} / c$, where $e$ is the electron charge, $b$ is the distance between the chains in the $\mathbf{b}$ direction, $v_{F}$ is the Fermi velocity, and $c$ is the speed of light. For the realistic values of the model parameters (see Sec. VIII), we estimate that $E_{H} / H \approx 1.8 \mathrm{~K} / \mathrm{T}$. The magnitude of the cyclotron energy, $E_{H} \approx 14 \mathrm{~K}$ at $H=7.8 \mathrm{~T}$, is close to the temperature of the resistivity minimum at that magnetic field, $T_{\min } \sim 20 \mathrm{~K}$. Taking into account that the minimum of resistivity clearly has a magnetic origin (it does not exist without magnetic field), and $T_{\min }$ grows with the increase of magnetic field, we suggest that the minimum of resistivity occurs when the temperature reaches the energy scale of the magnetic field; that is, $T_{\min } \approx E_{H}$.

Now we need to identify the nature of the second energy scale in the problem, the temperature of the resistivity maximum, $T_{\max }$. At the temperatures $T>T_{\max }$, it appears that $\rho_{a a}$ depends only on the magnetic field projection on the $\mathbf{c}^{*}$ axis perpendicular to the $\mathbf{a}$ and $\mathbf{b}$ directions 32 . From this observation, we may conclude that at $T>T_{\max }$ the system behaves effectively as a two-dimensional (2D) system; that is, the coupling between the chains in the $\mathbf{c}$ direction is not relevant. On the other hand, at $T<T_{\max }$ the coupling along the $\mathbf{c}$ axis becomes important. This is manifested by the magic angles effect, which is an essentially three-dimensional (3D) phenomenon involving both the $\mathbf{b}$ and $\mathbf{c}$ axes. The 
coupling between the chains along the $\mathbf{c}$ axis is characterized by the electron tunneling amplitude $t_{c}$, whose magnitude is believed to be of the order of $10 \mathrm{~K}$ [33], which is close to $T_{\max } \sim 8 \mathrm{~K}$. Thus, we suggest that the electron tunneling amplitude $t_{c}$ sets the temperature scale $T_{\max }$ of the resistivity maximum: $T_{\max } \approx t_{c}$. This conjecture is supported by the experimental fact that $T_{\max }\left(\right.$ unlike $T_{\min }$ ) does not depend appreciably on the magnetic field [3,4]. We also need to mention that, according to Refs. [34,35], the coupling between the chains in the $\mathbf{b}$ direction, $t_{b}$, is much greater than the temperatures discussed in our paper: $t_{b} \sim 300 \mathrm{~K}$

Taking into account these energy scales, we identify three qualitatively different regimes in the behavior of a Q1D system in a magnetic field:

1) High temperatures: $E_{H} \approx T_{\min }<T<t_{b}$. In this region, the temperature is greater than both the magnetic energy $E_{H}$ and the electron tunneling amplitude $t_{c}$ along the $\mathbf{c}$ axis, but lower than the tunneling amplitude $t_{b}$ along the $\mathbf{b}$ axis. Thus, we may neglect both the magnetic field and the coupling between the chains along the $\mathbf{c}$ axis and treat the system as a normal 2D Fermi liquid without magnetic field. This results in the quadratic law $\rho_{a a} \sim T^{2}$ and the metallic behavior of the resistivity $d \rho_{a a} / d T>0$.

2) Intermediate temperatures: $t_{c} \approx T_{\max }<T<T_{\min } \approx E_{H}$. In this region, the temperature is still greater than the coupling between the chains along the $\mathbf{c}$ axis, so the system remains 2D; however, the effect of the magnetic field becomes important. It is known that, in the presence of a magnetic field along the $\mathbf{c}$ axis, the motion of electrons along the $\mathbf{b}$ axis becomes quantized, and the dispersion law of electrons becomes one-dimensional (1D) [36]. The degeneracy of the electron spectrum in the $\mathbf{b}$ direction is a specific manifestation of the Landau degeneracy in a magnetic field in the case of a $2 \mathrm{D}$ system with a strong Q1D anisotropy. This phenomenon is called "one-dimensionalization" of a Q1D system by a magnetic field [11]. Even though the spectrum of electrons becomes 1D and their wave functions become localized in the $\mathbf{b}$ direction, the wave functions still spread over many chains (if $E_{H} \ll t_{b}$ ), which results in a considerable interaction between different chains 31. So the system is not truly 1D, because it does not consist of completely decoupled 1D 
chains. Nevertheless, we may expect that, at least, some 1D features would be present in this regime and, via a mechanism that need to be specified, would lead to an insulating transport behavior $d \rho_{a a} / d T<0$. In general, 1D systems have stronger tendency toward insulating behavior than higher-dimensional systems, because various insulating mechanisms, such as renormalization of umklapp scattering, density-wave instabilities, and Anderson localization, are more effective in one dimension than in higher dimensions. So the conjecture that the insulating behavior is caused by the magnetic-field-enforced "one-dimensionalization" is plausible, but requires detailed studying of a specific mechanism [1]. We review possible candidates for the mechanism in the next section and quantitatively analyze one of the mechanisms in rest of the paper.

3) Low temperatures: $T<T_{\max } \approx t_{c}$. In this region, the coupling between the $(\mathbf{a}, \mathbf{b})$ planes becomes important. The magnetic field pointing exactly along the $\mathbf{c}$ axis does not affect the electron motion along that axis. Thus, in addition to the magnetic-field-enforced 1D dispersion law discussed in part 2), the system acquired an extra dispersion in the c direction and becomes effectively 2D, which results in a metallic, Fermi-liquid behavior $d \rho_{a a} / d T>0$. If the magnetic field does not point along the $\mathbf{c}$ axis, the component of the field perpendicular to the c axis suppresses the energy dispersion along that axis, so the system remains effectively $1 \mathrm{D}$ and insulating: $d \rho_{a a} / d T<0$. If the direction of the field is close to the $\mathbf{c}$ axis, we expect resistivity to decrease with decreasing temperature in the range $E_{H}^{(c)}<T<T_{\max } \approx t_{c}$ and to start increasing again at $T<E_{H}^{(c)}$, where $E_{H}^{(c)}$ is the cyclotron energy of the electron motion along the $\mathbf{c}$ axis, which is proportional to the projection of the magnetic field perpendicular to the $\mathbf{c}$ axis. The same arguments apply not only to the $\mathbf{c}$ axis, but also to the $\mathbf{c}+\mathbf{b}$ axis and other integer crystallographic directions $m \mathbf{c}+n \mathbf{b}$. However, because the electron tunneling amplitudes in these directions decrease rapidly with the increase of the integers $m$ and $n$, the effect is clearly visible experimentally only for the $\mathbf{c}+\mathbf{b}$ axis.

In summary, we suggest that the unusual transport behavior of $(\mathrm{TMTSF})_{2} \mathrm{PF}_{6}$ results from the changes in the effective dimensionality of the system caused by the applied magnetic 
field. The system is $2 \mathrm{D}$ at $E_{H}<T<t_{b}$ and effectively $1 \mathrm{D}$ at $t_{c}<T<E_{H}$. At $T<t_{c}$ the system is effectively $2 \mathrm{D}$ for the magic orientations of the magnetic field and effectively $1 \mathrm{D}$ for generic orientations. Whenever the system is 2D (or 3D), it is a normal Fermi liquid, and the temperature dependence of resistivity is metallic. Whenever the system is effectively 1D, the temperature dependence of resistivity is insulating. The latter state of the system might be called the magnetic-field-induced Luttinger insulator (MFILI), by analogy with the term "Luttinger liquid", which refers to the metallic state of a 1D system [37.

We do not have detailed mathematical calculations that can prove the heuristic picture outlined in this section. Nevertheless, we can predict some experimental effects based on this picture. In Refs. 38, 34, oscillations of $\rho_{c c}$ upon rotation of a magnetic field in the (a, c) plane were discovered by Danner et al. Following the theoretical suggestion of Ref. [39], it was found that a small magnetic field along the $\mathbf{b}$ axis destroys the oscillations [34]. We predict that if a magnetic field is rotated in the magic plane from the $\mathbf{b}+\mathbf{c}$ direction toward the $\mathbf{a}$ direction, the Danner oscillations should exist, even though the magnetic field has a finite b-component. The suggested geometry has an advantage over the geometry of experiment [34], where the magnetic field had a fixed b-component, that the Danner oscillations would not be mixed up with the Lebed' oscillations occurring when the magnetic field is rotated in the $(\mathbf{b}, \mathbf{c})$ plane. This prediction is based on the idea that the Danner oscillations require that the electron motion in the third direction is not suppressed by the magnetic field, which happens only when the magnetic field belongs to a magic plane. We also predict that the Danner oscillations should disappear at $T>T_{\max }$, where the electron dispersion in the third direction is smeared out by temperature. A detailed study of magnetic oscillations upon rotation of the magnetic field around the $\mathbf{c}^{*}$ axis with different tilts relative to the $\mathbf{c}^{*}$ axis was recently performed by Lee and Naughton [40]. They found possible to interprete most, but not all, of the results within a conventional semiclassical theory of metals. 


\section{REVIEW OF THEORETICAL MODELS}

Within the heuristic framework presented in the previous Section, a theoretical study of the problem reduces to the following two parts:

(a) How a magnetic field induces the negative temperature dependence of resistivity $d \rho_{a a} / d T<0$ in a 2D metal with a strong Q1D anisotropy. (2D problem)

(b) How the electron tunneling in the third direction does or does not suppress the effect found in part (a) depending on the orientation of the magnetic field in the (b, c) plane. (3D problem)

From the above formulation, it is clear that the 3D problem (b) can be addressed only after the 2D problem (a) has been solved. In other words, we believe that the insulating temperature dependence of resistivity (problem (a)) and the drop of resistivity at the magic angles (problem (b)) have a common origin.

However, until recently, theoretical and experimental efforts were focused on solving problem (b) without recognizing and addressing problem (a). Soon after the experimental discovery of the magic angles [16 18], a number of theories tried to explain this effect semiclassically 414 43]. The theories [41,42] found the magic angles effect in $\rho_{b b}$ and $\rho_{c c}$, but not in $\rho_{a a}$. Microscopic analysis [29] of the "hot spots" model [43] demonstrated that it cannot explain the huge magnetoresistance and the magic angles effect. In all of these models, resistivity was calculated by introducing a relaxation time $\tau$ phenomenologically and studying semiclassical electron trajectories in the momentum space. These theories assumed that relaxation mechanism does not change dramatically as the magnetic field is rotated. In view of experiment [4], this assumption is completely wrong, because it is the switching between metallic and insulating states and the corresponding change in the relaxation mechanism that causes the magic angles effect.

Another explanation of the magic angles was proposed on the basis of the Luttinger liquid concept in Ref. [39] (see also Ref. 444). This theory suggested that the magic angles effect reflects the change of the effective dimensionality of the system: The system is a 2D 
Luttinger liquid at generic angles, and a 3D normal Fermi liquid at the magic angles. The prediction of the theory that even a small $\mathbf{b}$-component of the magnetic field would destroy coherence of the interchain hopping in the $\mathbf{c}$ direction was confirmed experimentally in Ref. [34,44]. However, this theory focuses only on problem (b), but does not address the issue of the temperature dependence of resistivity and does not explain how problem (a) may be solved. Moreover, in the actual calculations [39], $t_{c}$ is treated as a perturbation to a 1D system, and the much greater tunneling amplitude $t_{b}$ is effectively neglected. For this reason, the theory [39] actually studies the dimensionality crossover between $2 \mathrm{D}$ and $1 \mathrm{D}$, not $2 \mathrm{D}$ and $3 \mathrm{D}$, and the very important c-component of the magnetic field does not appear in these calculations.

The very first calculation of the angular dependence of $\rho_{a a}$ was done by Lebed' and Bak [20] before the experimental discovery of the magic angles. However, it predicted maxima, not minima, of resistance at the magic angles. This discrepancy was corrected in the subsequent work [45]. In this theory, resistivity $\rho_{a a} \propto 1 / \tau$ is studied by calculating the rate of umklapp scattering $1 / \tau$ in the lowest order of perturbation theory. This approach allows to study how resistivity depends on temperature and on the magnitude and orientation of the magnetic field. According to Ref. [45], the temperature dependence of the scattering rate changes with the magnetic field orientation: $1 / \tau \propto T^{2}$ at the magic angles and $1 / \tau \propto T$ at generic angles, because the electron dispersion law is 2D and 1D in these cases, respectively. At low enough temperatures, the difference between the $T^{2}$ and $T$ laws should result in sharp dips of resistance at the magic angles. However, the theory predicts that both temperature dependences are metallic $\left(d \rho_{a a} / d T>0\right)$, whereas experimentally the temperature dependence is insulating $\left(d \rho_{a a} / d T<0\right)$ for nonmagic angles at $T<T_{\min }$ and for all angles at $T_{\max }<T<T_{\min }$ [2 4 . Thus, the theory [45] is not adequate either.

In Ref. [20], Lebed' and Bak considered also the scattering rate of electrons on impurities renormalized by the electron-electron interaction in the lowest order and noted that it grows with decreasing temperature, when a magnetic field is applied. It is easy to check analytically that this scattering rate is approximately constant without magnetic field and, 
if $t_{c}$ is neglected, grows as $\ln \left(E_{H} / T\right)$ in a magnetic field at $T<E_{H}$. We calculated this diagram numerically in the 2D case and found that it increases by only about $20 \%$ in the relevant range of fields and temperatures, which is insufficient to explain the experiment. (Our numerical calculation of the umklapp electron-electron scattering diagram in the 2D case also confirmed that application of a magnetic field changes the temperature dependence of $1 / \tau$ from $T^{2}$ to $T$ at $T<E_{H}$, as discussed in the preceding paragraph.)

After the magnetic-field-induced insulating temperature dependence of $\rho_{a a}$ was discovered experimentally, theory started to address this problem specifically. Refs. [2.3] suggested that resistance increases at $T<T_{\min }$, because the "one-dimensionalization" of (TMTSF) ${ }_{2} \mathrm{X}$ by a magnetic field induces formation of a pseudogap in the charge channel. It is well known that charge and spin excitations are independent in a 1D system [12]. In the presence of umklapp scattering and repulsive interactions, charge excitations may develop a pseudogap, whereas spin excitations may remain gapless. This results in insulating temperature dependence of resistivity coexisting with metallic behavior of spin susceptibility. This effect is observed experimentally in the sulfur-based compounds (TMTTF $)_{2} \mathrm{X}$ [46], which are more 1D than the selenium-based compounds (TMTSF) ${ }_{2} \mathrm{X}$ (see Refs. [3]:99:[3]). Induction of a charge pseudogap by a magnetic field would explain insulating behavior of all components of the resistivity tensor in (TMTSF $)_{2} \mathrm{PF}_{6}$ [22 25] and the NMR data in (TMTSF) ${ }_{2} \mathrm{ClO}_{4}$ [2] (although the transverse resistivity in (TMTSF) ${ }_{2} \mathrm{ClO}_{4}$ behaves differently [27,28]).

However, in order to achieve quantitative agreement with the experiment, this theory assumes that $t_{b} \sim 50 \mathrm{~K}[2]$ or 15-30 $\mathrm{K}$ [国], which is almost an order of magnitude smaller that the commonly accepted value $t_{b} \approx 250 \mathrm{~K}$ deduced from the $\mathbf{b}$-axis plasma edge [35] and the $(\mathbf{a}, \mathbf{b})$-plane angular magnetic oscillation [34]. In this theory, the magnitude of the pseudogap is determined by the energy scale where the renormalization-group (RG) equations (also called the parquet equations [47]) for the forward, backward, and umklapp scattering amplitudes of electron-electron interaction diverge. However, because the chains are strongly coupled, it is not correct to limit the RG equations to only those three amplitudes of interaction. It is necessary to include an infinite number of the amplitudes of interaction 
between different chains, which makes the RG equations integro-differential [48]. For a Q1D conductor in a magnetic field, the integro-differential RG equations were derived and solved numerically without umklapp in Refs. [31,49, 50]. The temperature where a solution of the RG equations diverges was interpreted in the latter papers as the FISDW transition temperature $T_{c}$. On the other hand, in a purely $1 \mathrm{D}$ case, the energy where a solution of the RG equations diverges is conventionally interpreted as a pseudogap energy, not as a transition temperature, because thermodynamic phase transitions are not possible in 1D systems [51. Thus, the problem is whether the RG equations for a Q1D conductor in a magnetic field can simultaneously describe formation of a pseudogap at a high temperature and the FISDW transition at a much lower temperature. In a purely $1 \mathrm{D}$ case this is possible, because the $\mathrm{RG}$ equations separate exactly into two independent sets of equations for the spin and charge channels [47]. However, in a higher-dimensional case, all of the interaction amplitudes are coupled, and separation of the RG equations into independent channels does not seem feasible. It is not clear why a pseudogap energy and a FISDW transition temperature would differ by an order of magnitude in the RG approach, if both are produced by the same mechanism of "one-dimensionalization" and enhancement of the Peierls susceptibility by a magnetic field.

Even after the RG equations are solved, the temperature dependence of resistivity still needs to be calculated. This issue was addressed in the theory by Gor'kov [52, 53], which suggested that resistivity is given by the same diagram that was studied in Refs. [20,45], but with a renormalized, temperature-dependent umklapp scattering amplitude. In the standard model of the FISDW transition [54], where $t_{b} \gg E_{H}$, and electron-electron interaction is repulsive, it is safe to neglect the superconducting channel contribution to the RG equations [31]. In this case, the RG equations reduce to the conventional ladder-RPA equations. The umklapp scattering amplitude, renormalized according to these equations, grows when $T \rightarrow T_{c}$. Because $\rho_{a a}$ is proportional to the square of the umklapp amplitude, the insulating regime $d \rho_{a a} / d T<0$ might be achieved if the umklapp amplitude grows faster than the phase space factor $T$ or $T^{2}$ decreases. One might expect, though, that resistivity would 
start to grow only in a narrow vicinity of the FISDW transition, not at the temperatures an order of magnitude higher than $T_{c}$. Whether this approach can quantitatively explain the experiment, particularly the role of a magnetic field, can be verified by numerical calculations. In the rest of the paper, we study this issue in detail. The approach of Refs. [52,53] is attractive, because it permits straightforward calculation of transport coefficients and connects naturally with the standard, successful model of FISDW on one hand and with the simpler, better-understood transport model of Refs. [20,45] on the other hand.

\section{Q1D CONDUCTOR IN A MAGNETIC FIELD}

For the following theoretical description, we select the $x, y$, and $z$ axes along the crystal directions $\mathbf{a}, \mathbf{b}$, and $\mathbf{c}$, which are not orthogonal in the triclinic (TMTSF) ${ }_{2} \mathrm{X}$ crystals. Electrons can tunnel between different chains with the amplitudes $t_{\mathbf{l}}$, where $\mathbf{l}=\left(l_{1}, l_{2}\right)$ is a $2 \mathrm{D}$ integer vector that determines the transverse displacement of the electron, $\mathbf{d}_{\mathbf{l}}=l_{1} \mathbf{b}+l_{2} \mathbf{c}$, in the process of tunneling. The Fermi surface of $1 \mathrm{D}$ electron motion along the chains consists of two Fermi points characterized by the Fermi wave vectors $\pm k_{F}$. We label the electrons with the wave vectors close to $\pm k_{F}$ by the index $\alpha= \pm$. In the vicinity of the Fermi energy, the energy dispersion law of the longitudinal electron motion can be linearized as $\varepsilon= \pm \hbar v_{F} k_{x}$, where $\hbar$ is the Planck constant, $v_{F}$ is the Fermi velocity, the energy $\varepsilon$ is counted from the Fermi energy, and the longitudinal wave vector $k_{x}$ is counted from $\pm k_{F}$ for the two Fermi points. In the absence of magnetic field, the total, longitudinal and transverse, electron dispersion law is

$$
\varepsilon_{\alpha}(\mathbf{k})=\alpha \hbar v_{F} k_{x}+\sum_{\mathbf{l}} 2 t_{\mathbf{l}} \cos \left(\mathbf{k}_{\perp} \mathbf{d}_{\mathbf{l}}+\alpha \varphi_{\mathbf{l}}\right)
$$

where $\mathbf{k}=\left(k_{x}, k_{y}, k_{z}\right)=\left(k_{x}, \mathbf{k}_{\perp}\right)$ are the electron wave vectors along the $x, y$, and $z$ axes. The phases $\varphi_{1}$ in the transverse dispersion law (1) of a triclinic crystal are determined by the amplitudes of tunneling to different molecules belonging to the same chain [56]. In this

paper, we present analytical formulas for a general dispersion law (1) with any number of 
the transverse tunneling amplitudes $t_{1}$, but we perform numerical calculations only for the $2 \mathrm{D}$ case with the two tunneling amplitudes: $t_{b}=t_{1,0}$ between the nearest and $t_{b}^{\prime}=t_{2,0}$ between the next-nearest chains in the $\mathbf{b}$ direction. The values of the corresponding phases, $\varphi_{b}$ and $\varphi_{b}^{\prime}$, are not known reliably. According to Yamaji [56], $\varphi_{b}^{\prime}=-\pi / 2$, and $\varphi_{b}$ varies from $7^{\circ}$ to $40^{\circ}$ when temperature varies from $300 \mathrm{~K}$ to $1.7 \mathrm{~K}$ in (TMTSF) ${ }_{2} \mathrm{PF}_{6}$. In our numerical calculations, we assume $\varphi_{b}^{\prime}=2 \varphi_{b}$ for simplicity and consider several values of $\varphi_{b}$ between the two extremal values $\varphi_{b}=0$ and $\varphi_{b}=\pi / 4$.

Suppose that a magnetic field $\mathbf{H}$ is applied perpendicular to the chains. It can be introduced into the Hamiltonian of the system via the Peierls-Onsager substitution $\mathbf{k} \rightarrow$ $\mathbf{k}-x\left[\mathbf{H} \times \mathbf{e}_{x}\right] e / \hbar C$, where $\mathbf{e}_{x}$ is the unit vector along the $x$ axis, $e$ is the electron charge, and $C$ is the speed of light. The eigenfunctions $\psi_{\alpha, \mathbf{k}}$ of noninteracting electrons in the magnetic field $H$ are 31$]$

$\psi_{\alpha, \mathbf{k}}\left(x, n_{y}, n_{z}\right)=\frac{1}{\sqrt{L \mathcal{N}}} \exp \left[i\left(k_{x} x+k_{y} n_{y} b+k_{z} n_{z} c+\alpha \sum_{\mathbf{l}} \frac{2 t_{\mathbf{l}}}{\hbar v_{F} G_{\mathbf{l}}} \sin \left(\mathbf{k}_{\perp} \mathbf{d}_{\mathbf{l}}-G_{\mathbf{l}} x+\alpha \varphi_{\mathbf{l}}\right)\right)\right]$,

where $G_{\mathbf{l}}=\mathbf{e}_{x} \cdot\left[\mathbf{d}_{\mathbf{l}} \times \mathbf{H}\right] e / \hbar C$ are the wave vectors proportional to the magnetic field, $n_{y}$ and $n_{z}$ are the integer coordinates of the chains in the $y$ and $z$ directions, $L$ is the length of a chain, and $\mathcal{N}$ is the total number of the chains. The eigenenergies of eigenfunctions (2) are

$$
\varepsilon=\alpha \hbar v_{F} k_{x}
$$

thus the electron dispersion law is effectively 1D in the magnetic field.

The single-particle Green function of noninteracting electrons in the magnetic field $H$ was found in Ref. 36:

$$
\begin{aligned}
& \mathcal{G}_{\alpha}\left(x, x^{\prime}, \mathbf{k}_{\perp}, i \omega_{m}\right)=\int_{-\infty}^{\infty} \frac{d k_{x}}{2 \pi} \frac{\exp \left[i k_{x}\left(x-x^{\prime}\right)\right]}{i \omega_{m}-\alpha \hbar v_{F} k_{x}} \\
& \times \exp \left(i \alpha \sum_{\mathbf{l}} \frac{2 t_{\mathbf{l}}}{\hbar v_{F} G_{\mathbf{l}}}\left[\sin \left(\mathbf{k}_{\perp} \mathbf{d}_{\mathbf{l}}-G_{\mathbf{l}} x+\alpha \varphi_{\mathbf{l}}\right)-\sin \left(\mathbf{k}_{\perp} \mathbf{d}_{\mathbf{l}}-G_{\mathbf{l}} x^{\prime}+\alpha \varphi_{\mathbf{l}}\right)\right]\right),
\end{aligned}
$$


where $\omega_{m}=2 \pi(m+1 / 2) T$ is the Matsubara frequency. The Green function (4) is a product of two terms: The first term is the Green function of 1D electrons, whereas the second, exponential term represents the transverse motion of the electrons. Only the second term contains the magnetic field via the wave vectors $G_{1}$, which introduce periodic dependences on $x$ and make the Green function (4) not translationally invariant along the chains. In 2D case, we denote the magnetic wave vector $G_{1,0}$ as simply $G$. The integral over $k_{x}$ in Eq. (四) can be easily taken, but the Lehmann representation (四) is more convenient for analytic continuation from the Matsubara frequencies to the real frequencies.

\section{RENORMALIZATION OF THE UMKLAPP SCATTERING AMPLITUDE DUE TO SDW INSTABILITY}

The tendency of a Q1D system toward the SDW instability manifests itself in divergence of the density-wave susceptibility, which is shown diagrammatically in the lowest order in Fig. 1. In this figure, the solid and dashed lines represent the Green functions of the + and - electrons, and $\Omega_{m}$ and $\mathbf{q}_{\perp}$ are the incoming energy and the transverse wave vector. In the Matsubara technique, the analytic expression for the bare susceptibility per one chain is 36]

$$
\begin{aligned}
\chi_{0}\left(x^{\prime}-x, \mathbf{q}_{\perp}, i \Omega_{m}\right) & =-T \sum_{j} \int \frac{b c d^{2} \mathbf{k}_{\perp}}{(2 \pi)^{2}} G_{-}\left(x, x^{\prime}, \mathbf{k}_{\perp}+\mathbf{q}_{\perp}, i \omega_{j}+i \Omega_{m}\right) G_{+}\left(x^{\prime}, x, \mathbf{k}_{\perp}, i \omega_{j}\right) \\
& =\frac{T}{2 \pi\left(\hbar v_{F}\right)^{2}} \frac{\lambda\left(x^{\prime}-x, \mathbf{q}_{\perp}\right)}{\sinh \left[2 \pi T\left(x^{\prime}-x\right) / \hbar v_{F}\right]} \int_{-\infty}^{\infty} \frac{d \varpi \sin \left[\left(x^{\prime}-x\right) \varpi / \hbar v_{F}\right]}{\varpi-i \Omega_{m}}
\end{aligned}
$$

where

$$
\lambda\left(x, \mathbf{q}_{\perp}\right)=\int \frac{b c d \mathbf{k}_{\perp}}{(2 \pi)^{2}} \exp \left(-i \sum_{\mathbf{l}} \frac{8 t_{\mathbf{l}}}{\hbar v_{F} G_{\mathbf{l}}} \sin \left(G_{\mathbf{l}} x / 2\right) \cos \left(\mathbf{k}_{\perp} \mathbf{d}_{\mathbf{l}}\right) \cos \left(\mathbf{q}_{\perp} \mathbf{d}_{\mathbf{l}} / 2-\varphi_{\mathbf{l}}\right)\right)
$$

In Eqs. (5) and (6) and elsewhere, the integration over the transverse wave vectors $\mathbf{k}_{\perp}$ goes over the Brillouin zone. Because of the averaging over $\mathbf{k}_{\perp}$, susceptibility (5) is a translationally invariant function of $x^{\prime}-x$, unlike the Green function (4). This allows us to Fourier-transform Eq. (5) over $x^{\prime}-x$ and to obtain $\chi_{0}\left(\mathbf{q}, i \Omega_{m}\right)$ as a function of the $3 \mathrm{D}$ 
wave vector $\mathbf{q}$. As follows from Eq. (5), $\chi_{0}\left(x, \mathbf{q}_{\perp}, i \Omega_{m}\right)$ behaves as $1 / x$ when $x \leq v_{F} / 2 \pi T$. This results in logarithmical divergence of $\chi_{0}\left(\mathbf{q}, i \Omega_{m}\right)$, which we cut off at a small distance $x_{0}=1 / 2 \gamma k_{F}$ [54], where $\gamma$ is the Euler constant [55].

Because the electron conduction band in (TMTSF $)_{2} \mathrm{X}$ is half-filled, the Fermi wave vector is commensurate with the crystal lattice wave vector along the chains: $4 k_{F}=2 \pi / a$. This relation permits the umklapp scattering process, where two "+" electrons are transformed into two "-" electrons, and the change of the total electron wave vector, $4 k_{F}$, is absorbed into the lattice wave vector $2 \pi / a$. The amplitude of this process is conventionally denoted by $\gamma_{3}$ [47. The one-loop diagram of Fig. 1 generates a ladder renormalization of the vertices of interaction between electrons as shown diagrammatically in Fig. 2. In this figure, the wavy lines represent the bare, unrenormalized vertices of forward $\left(g_{2}\right)$ and umklapp $\left(g_{3}\right)$ scattering, the circles represent the corresponding renormalized vertices $\gamma_{2}$ and $\gamma_{3}$, and the thin lines inside the circles indicate spin conservations along the electron lines. Appropriate for the SDW channel, the + and - electron lines in Fig. 2 have opposite spins. The equations of Fig. 2 are the same as in Ref. [53]. The analytic expression of these equations is

$$
\begin{aligned}
& \gamma_{2}\left(\mathbf{q}, i \Omega_{m}\right)=g_{2}+g_{2} \chi_{0}\left(\mathbf{q}, i \Omega_{m}\right) \gamma_{2}\left(\mathbf{q}, i \Omega_{m}\right)+g_{3} \chi_{0}\left(-\mathbf{q},-i \Omega_{m}\right) \gamma_{3}\left(\mathbf{q}, i \Omega_{m}\right) \\
& \gamma_{3}\left(\mathbf{q}, i \Omega_{m}\right)=g_{3}+g_{2} \chi_{0}\left(-\mathbf{q},-i \Omega_{m}\right) \gamma_{3}\left(\mathbf{q}, i \Omega_{m}\right)+g_{3} \chi_{0}\left(\mathbf{q}, i \Omega_{m}\right) \gamma_{2}\left(\mathbf{q}, i \Omega_{m}\right)
\end{aligned}
$$

and the solution is

$$
\begin{aligned}
\gamma_{2}\left(\mathbf{q}, i \Omega_{m}\right) & =\frac{g_{2}-\left(g_{2}^{2}-g_{3}^{2}\right) \chi_{0}\left(-\mathbf{q},-i \Omega_{m}\right)}{\left[1-g_{2} \chi_{0}\left(\mathbf{q}, i \Omega_{m}\right)\right]\left[1-g_{2} \chi_{0}\left(-\mathbf{q},-i \Omega_{m}\right)\right]-g_{3}^{2} \chi_{0}\left(\mathbf{q}, i \Omega_{m}\right) \chi_{0}\left(-\mathbf{q},-i \Omega_{m}\right)} \\
\gamma_{3}\left(\mathbf{q}, i \Omega_{m}\right) & =\frac{g_{3}}{\left[1-g_{2} \chi_{0}\left(\mathbf{q}, i \Omega_{m}\right)\right]\left[1-g_{2} \chi_{0}\left(-\mathbf{q},-i \Omega_{m}\right)\right]-g_{3}^{2} \chi_{0}\left(\mathbf{q}, i \Omega_{m}\right) \chi_{0}\left(-\mathbf{q},-i \Omega_{m}\right)} .
\end{aligned}
$$

Notice that whenever the umklapp vertex appears in the r.h.s. of Eqs. (17) and (8), the signs of $\mathbf{q}$ and $i \Omega_{m}$ in the argument of $\chi_{0}$ are reversed.

As temperature decreases, the bare susceptibility $\chi_{0}(\mathbf{q}, 0)$ grows until the denominator in Eqs. (9) and (10) vanishes at a certain temperature $T_{c}$, which is the FISDW transition temperature:

$$
\left[1-g_{2} \chi_{0}(\mathbf{q}, 0)\right]\left[1-g_{2} \chi_{0}(-\mathbf{q}, 0)\right]-g_{3}^{2} \chi_{0}(\mathbf{q}, 0) \chi_{0}(-\mathbf{q}, 0)=0
$$


The interaction amplitudes $\gamma_{2,3}(\mathbf{q}, 0)$ diverge at the transition temperature. Since Eq. (11) is a quadratic form of $\chi_{0}(\mathbf{q}, 0)$ and $\chi_{0}(-\mathbf{q}, 0)$, it vanishes at two different temperatures for a given value of $\mathbf{q}$. Usually, it is assumed that only the higher temperature is physically significant, and the transition temperature is determined from Eq. (11) by selecting the wave vector $\mathbf{q}$ that provides the maximal value for $T_{c}$.

By considering equations similar to Eq. (11), Lebed' [57] predicted that the umklapp splitting of the FISDW instability would result in oscillations of $T_{c}$ vs $H$, but the effect was not observed experimentally. On the other hand, the experimental $T$ - $H$ phase diagram of (TMTSF $)_{2} \mathrm{PF}_{6}$ can be well reproduced while neglecting umklapp [54. In order not to spoil the phase diagram by the umklapp splitting and to avoid unnecessary complications, we assume that $g_{3}$ is sufficiently small and neglect it in Eq. (11) and in the denominator of Eq. (10) 58]. Using the conventional band-structure parameters of (TMTSF) ${ }_{2} \mathrm{PF}_{6}: t_{a}=2900 \mathrm{~K}$ $\left(E_{F}=\sqrt{2} t_{a}\right), t_{b}=t_{a} / 10$, and $v_{F}=2 \times 10^{5} \mathrm{~m} / \mathrm{s}$, we find that the choice $t_{b}^{\prime}=20 \mathrm{~K}$ and $\tilde{g}_{2}=$ $g_{2} / 2 \pi \hbar v_{F}=0.2288$ produces a $T-H$ phase diagram close to the one observed experimentally in Ref. [59] at 12 kbar. The phase diagram does not depend on $\varphi_{b}$ and is shown in Fig. 3. In this figure, various symbols indicate the integer number $N$ of the quantized longitudinal wave vector of FISDW: $q_{x}=N G$ [54]. Note that wide spacing in $H$ between the points of our calculations would not allow us to observe the oscillations of $T_{c}$ vs $H$ [57], even if we took into account the umklapp splitting of the FISDW instability. If we set $t_{b}^{\prime}=0$, the electron spectrum (11) acquires perfect nesting: $\varepsilon_{+}\left(k_{x}, k_{y}\right)=-\varepsilon_{-}\left(k_{x}, k_{y}-\left(\pi-2 \varphi_{b}\right) / b\right)$, and the SDW transition temperature $T_{0}=\left(\hbar v_{F} / \pi x_{0}\right) \exp \left(-1 / \tilde{g}_{2}\right)=14.7 \mathrm{~K}$ becomes independent of the magnetic field.

While the Matsubara representation of $\gamma_{2,3}$ is useful for determining the $T$ - $H$ phase diagram, we need the scattering vertices at the real frequencies to calculate resistivity. Analytically continuing Eq. (5) from the Matsubara frequencies $i \Omega_{m}$ to the real energies $\varepsilon$, we find the following expression for the bare susceptibility:

$$
\chi_{0}(\mathbf{q}, \varepsilon)=\frac{T}{\left(\hbar v_{F}\right)^{2}} \int_{x_{0}}^{\infty} d x \frac{\operatorname{Re}\left\{\exp \left(-i q_{x} x\right) \lambda\left(x, \mathbf{q}_{\perp}\right)\right\} \exp \left(i x \varepsilon / \hbar v_{F}\right)}{\sinh \left(2 \pi T x / \hbar v_{F}\right)}
$$


Substituting Eq. (12) into Eqs. (9) and (10), we find the scattering vertices $\gamma_{2,3}(\mathbf{q}, \varepsilon)$ at the real energies $\varepsilon$.

\section{UMKLAPP RESISTIVITY OF A Q1D METAL}

Using the variational principle for the Boltzmann equation [60], we find the following expression for the resistivity along the chains due to electron-electron umklapp scattering:

$$
\rho_{x x}=\frac{2(\pi \hbar)^{2} b c}{e^{2} T L \mathcal{N}} \sum_{\mathbf{k}_{1}, \mathbf{k}_{2}, \mathbf{k}_{3}, \mathbf{k}_{4}} f_{0}\left(\varepsilon_{1}\right) f_{0}\left(\varepsilon_{2}\right)\left[1-f_{0}\left(\varepsilon_{3}\right)\right]\left[1-f_{0}\left(\varepsilon_{4}\right)\right] W_{\mathbf{k}_{1}, \mathbf{k}_{2}}^{\mathbf{k}_{3}, \mathbf{k}_{4}},
$$

where $\varepsilon_{i}=\varepsilon\left(\mathbf{k}_{i}\right)$ are the energies of electrons in the eigenstates $\left|\mathbf{k}_{i}\right\rangle(2), f_{0}(\varepsilon)$ is the Fermi distribution function, and $W_{\mathbf{k}_{1}, \mathbf{k}_{2}}^{\mathbf{k}_{3}, \mathbf{k}_{4}}$ is the scattering rate of two electrons from the states $\left|\mathbf{k}_{1}\right\rangle$ and $\left|\mathbf{k}_{2}\right\rangle$ into the states $\left|\mathbf{k}_{3}\right\rangle$ and $\left|\mathbf{k}_{4}\right\rangle$ :

$$
W_{\mathbf{k}_{1}, \mathbf{k}_{2}}^{\mathbf{k}_{3}, \mathbf{k}_{4}}=\frac{2 \pi}{\hbar}\left|\left\langle\mathbf{k}_{1}, \mathbf{k}_{2}\left|\gamma_{3}\right| \mathbf{k}_{3}, \mathbf{k}_{4}\right\rangle\right|^{2} \delta\left(\varepsilon_{1}+\varepsilon_{2}-\varepsilon_{3}-\varepsilon_{4}\right)
$$

Here $\delta(\varepsilon)$ is the Dirac delta function, and the matrix element is

$$
\begin{aligned}
& \left\langle\mathbf{k}_{1}, \mathbf{k}_{2}\left|\gamma_{3}\right| \mathbf{k}_{3}, \mathbf{k}_{4}\right\rangle=\sum_{n_{y}^{(1)}, n_{y}^{(2)}, n_{z}^{(1)}, n_{z}^{(2)}} \int_{-\infty}^{\infty} d x_{1} d x_{2} \psi_{+, \mathbf{k}_{1}}\left(x_{1}, n_{y}^{(1)}, n_{z}^{(1)}\right) \psi_{+, \mathbf{k}_{2}}\left(x_{2}, n_{y}^{(2)}, n_{z}^{(2)}\right) \\
& \times \psi_{-, \mathbf{k}_{3}}^{*}\left(x_{2}, n_{y}^{(2)}, n_{z}^{(2)}\right) \psi_{-, \mathbf{k}_{4}}^{*}\left(x_{1}, n_{y}^{(1)}, n_{z}^{(1)}\right) \gamma_{3}\left(x_{1}-x_{2}, n_{y}^{(1)}-n_{y}^{(2)}, n_{z}^{(1)}-n_{z}^{(2)}, \varepsilon_{2}-\varepsilon_{3}\right) .
\end{aligned}
$$

The vertex of interaction $\gamma_{3}$ is written in the mixed, coordinate and energy, representation, describing the umklapp scattering of the two "+" electrons with the coordinates $x_{1}$ and $x_{2}$

located on the chains $\left(n_{1}^{(y)}, n_{1}^{(z)}\right)$ and $\left(n_{2}^{(y)}, n_{2}^{(z)}\right)$ from the states $\left|\mathbf{k}_{1}\right\rangle$ and $\left|\mathbf{k}_{2}\right\rangle$ into the two "_" states $\left|\mathbf{k}_{3}\right\rangle$ and $\left|\mathbf{k}_{4}\right\rangle$ with the same coordinates.

Substituting Eq. (2) into Eq. (15) and changing the variables of integration $k_{x}^{(i)} \rightarrow \varepsilon_{i}$ via Eq. (3), we find the following expression for the resistivity

$$
\rho_{x x}=\frac{2(\pi \hbar)^{2} L^{3} b c}{e^{2} T \mathcal{N}} \int_{-\infty}^{\infty} \frac{d \varepsilon_{1} d \varepsilon_{2} d \varepsilon_{3} d \varepsilon_{4}}{\left(2 \pi \hbar v_{F}\right)^{4}} f_{0}\left(\varepsilon_{1}\right) f_{0}\left(\varepsilon_{2}\right)\left[1-f_{0}\left(\varepsilon_{3}\right)\right]\left[1-f_{0}\left(\varepsilon_{4}\right)\right] W_{\varepsilon_{1}, \varepsilon_{2}}^{\varepsilon_{3}, \varepsilon_{4}},
$$

where 


$$
\begin{aligned}
& W_{\varepsilon_{1}, \varepsilon_{2}}^{\varepsilon_{3}, \varepsilon_{4}}=\sum_{\mathbf{k}_{\perp}^{(1)}, \mathbf{k}_{\perp}^{(2)}, \mathbf{k}_{\perp}^{(3)}, \mathbf{k}_{\perp}^{(4)}} W_{\mathbf{k}_{1}, \mathbf{k}_{2}}^{\mathbf{k}_{3}, \mathbf{k}_{4}} \\
& =\frac{b c \mathcal{N}}{\hbar L^{3}} \int_{-\infty}^{\infty} d x^{\prime} d x^{\prime \prime} \int \frac{d^{3} \mathbf{q}}{(2 \pi)^{2}}\left|\gamma_{3}\left(\mathbf{q}, \varepsilon_{2}-\varepsilon_{3}\right)\right|^{2} \delta\left(\varepsilon_{1}+\varepsilon_{2}-\varepsilon_{3}-\varepsilon_{4}\right) \\
& \times \exp \left[i\left(q_{x}+\frac{\varepsilon_{1}+\varepsilon_{4}}{\hbar v_{F}}\right) x^{\prime}\right] \lambda\left(x^{\prime},-\mathbf{q}_{\perp}\right) \exp \left[i\left(-q_{x}+\frac{\varepsilon_{2}+\varepsilon_{3}}{\hbar v_{F}}\right) x^{\prime \prime}\right] \lambda\left(x^{\prime \prime}, \mathbf{q}_{\perp}\right) .
\end{aligned}
$$

Using the $\delta$-function from Eq. (17), we take the integral over $\varepsilon_{4}$ in Eq. (16). Then, changing the integration variables $\varepsilon_{1}, \varepsilon_{2}$, and $\varepsilon_{3}$ to $\varepsilon=\varepsilon_{2}-\varepsilon_{3}, \varepsilon_{1}^{\prime}=\varepsilon_{1}+\left(\varepsilon_{2}-\varepsilon_{3}\right) / 2$, and $\varepsilon_{2}^{\prime}=\varepsilon_{2}+\varepsilon_{3}$ and taking the integrals over $\varepsilon_{1}^{\prime}$ and $\varepsilon_{2}^{\prime}$, we obtain the final expression for resistivity:

$$
\rho_{x x}=\frac{\hbar(b c)^{2}}{32 e^{2} T} \int_{-\infty}^{\infty} d \varepsilon \int d^{3} \mathbf{q} F(\mathbf{q}, \varepsilon) F(-\mathbf{q}, \varepsilon)\left|\tilde{\gamma}_{3}(\mathbf{q}, \varepsilon)\right|^{2}
$$

where

$$
\begin{aligned}
& F(\mathbf{q}, \varepsilon)=\frac{2 T}{\hbar v_{F} \sinh (\varepsilon / 2 T)} \int_{-\infty}^{\infty} d x \frac{\exp \left(-i q_{x} x\right) \lambda\left(x, \mathbf{q}_{\perp}\right) \sin \left(x \varepsilon / \hbar v_{F}\right)}{\sinh \left(2 \pi T x / \hbar v_{F}\right)} \\
& \tilde{\gamma}_{3}(\mathbf{q}, \varepsilon)=\frac{\gamma_{3}(\mathbf{q}, \varepsilon)}{2 \pi \hbar v_{F}}
\end{aligned}
$$

are dimensionless functions. The renormalized umklapp vertex $\gamma_{3}(\mathbf{q}, \varepsilon)$, given by Eqs. (10) and (12), should be substituted into Eqs. (18) and (20). If the renormalization of $\gamma_{3}$ is neglected $\left(\gamma_{3}=g_{3}\right)$, the integral over $\varepsilon$ in Eq. (18) can be taken analytically, and the result agrees with the expressions obtained in Refs. [20,45].

\section{TEMPERATURE DEPENDENCE OF RESISTIVITY IN ZERO MAGNETIC FIELD}

Having obtained the general expression (18) for the longitudinal resistivity, let us examine the limit of zero magnetic field first. In this case, $F(\mathbf{q}, \varepsilon)(19)$ becomes

$$
F(\mathbf{q}, \varepsilon)=\frac{b c}{(2 \pi)^{2}} \int \frac{d^{2} \mathbf{k}_{\perp}}{\cosh \left(\frac{\varepsilon}{2 T}\right)+\cosh \left(\frac{\hbar v_{F} q_{x}+\sum_{\mathbf{l}} 4 t_{\mathbf{l}} \cos \left(\mathbf{q}_{\perp} \mathbf{d}_{\mathbf{l}} / 2-\varphi_{\mathbf{l}}\right) \cos \left(\mathbf{k}_{\perp} \mathbf{d}_{\mathbf{l}}\right)}{2 T}\right)} .
$$

The function $F(\mathbf{q}, \varepsilon)(21)$, confines integration in Eq. (18) to the energy interval

$$
|\varepsilon| \lesssim T
$$


and to the wave-vectors region defined by the two inequalities:

$$
\begin{gathered}
\left|\hbar v_{F} q_{x}+\sum_{\mathbf{l}} 4 t_{\mathbf{l}} \cos \left(\mathbf{q}_{\perp} \mathbf{d}_{\mathbf{l}} / 2-\varphi_{\mathbf{l}}\right) \cos \left(\mathbf{k}_{\perp} \mathbf{d}_{\mathbf{l}}\right)\right| \lesssim T \\
\left|\hbar v_{F} q_{x}-\sum_{\mathbf{l}} 4 t_{\mathbf{l}} \cos \left(\mathbf{q}_{\perp} \mathbf{d}_{\mathbf{l}} / 2+\varphi_{\mathbf{l}}\right) \cos \left(\mathbf{k}_{\perp}^{\prime} \mathbf{d}_{\mathbf{l}}\right)\right| \lesssim T .
\end{gathered}
$$

It follows from Eqs. (23) and (24) that the integration over the transverse wave vectors is restricted by the inequality

$$
\left|\Xi\left(\mathbf{q}_{\perp}, \mathbf{k}_{\perp}, \mathbf{k}_{\perp}^{\prime}\right)\right| \lesssim T
$$

where

$$
\Xi\left(\mathbf{q}_{\perp}, \mathbf{k}_{\perp}, \mathbf{k}_{\perp}^{\prime}\right)=\sum_{\mathbf{l}} 4 t_{\mathbf{l}}\left[\cos \left(\mathbf{q}_{\perp} \mathbf{d}_{\mathbf{l}} / 2-\varphi_{\mathbf{l}}\right) \cos \left(\mathbf{k}_{\perp} \mathbf{d}_{\mathbf{l}}\right)+\cos \left(\mathbf{q}_{\perp} \mathbf{d}_{\mathbf{l}} / 2+\varphi_{\mathbf{l}}\right) \cos \left(\mathbf{k}_{\perp}^{\prime} \mathbf{d}_{\mathbf{l}}\right)\right]
$$

The integrals over $\varepsilon$ and $q_{x}$ in Eq. (18) with the function $F(\mathbf{q}, \varepsilon)$ (21) can be taken analytically, provided we neglect dependence of $\gamma_{3}\left(q_{x}, \mathbf{q}_{\perp}, \varepsilon\right)$ on $q_{x}$ and $\varepsilon$, that is, replace $\gamma_{3}\left(q_{x}, \mathbf{q}_{\perp}, \varepsilon\right)$ by $\gamma_{3}\left(\bar{q}_{x}, \mathbf{q}_{\perp}, \bar{\varepsilon}\right)$, where $\bar{q}_{x}$ and $\bar{\varepsilon}$ are some characteristic values of $q_{x}$ and $\varepsilon$ from the intervals of integration (22), (23), and (24):

$$
\rho_{x x} \approx \frac{\pi^{2}(b c)^{4} T}{16 e^{2} v_{F}} \int \frac{d^{2} \mathbf{q}_{\perp} d^{2} \mathbf{k}_{\perp} d^{2} \mathbf{q}_{\perp}^{\prime}}{(2 \pi)^{6}}\left(\frac{\Xi\left(\mathbf{q}_{\perp}, \mathbf{k}_{\perp}, \mathbf{k}_{\perp}^{\prime}\right) / T}{\sinh \left[\Xi\left(\mathbf{q}_{\perp}, \mathbf{k}_{\perp}, \mathbf{k}_{\perp}^{\prime}\right) / 4 T\right]}\right)^{2}\left|\tilde{\gamma}_{3}\left(\bar{q}_{x}, \mathbf{q}_{\perp}, \bar{\varepsilon}\right)\right|^{2} .
$$

This approximation should be valid provided the peak in $\gamma_{3}\left(q_{x}, \mathbf{q}_{\perp}, \varepsilon\right)$ at $\varepsilon=0$ and $\mathbf{q}$ equal to the nesting vector is wider than temperature, which is the case for temperatures not very close to the transition temperature $T_{c}$. Eq. (27) with $\Xi(26)$ coincides with the expression obtained by Gor'kov [52,53] for $\varphi_{1}=0$, except that we find an additional factor $1 / 2$ in the argument of sinh, as in Ref. [61].

If renormalization of $\gamma_{3}$ is neglected, and $\gamma_{3}$ is replaced by a constant $g_{3}$, then integral (27) gives the volume of the wave-vectors space restricted by inequality (25). In a general $2 \mathrm{D}$ or $3 \mathrm{D}$ case, this volume is proportional to $T$, thus resistance (27) is proportional to $T^{2}$ :

$$
\rho_{x x}^{(2 \mathrm{D}, 3 \mathrm{D})} \propto \tilde{g}_{3}^{2} T^{2}
$$

which is the standard result of the Fermi-liquid theory [14. In $1 \mathrm{D}$ case [62, where $\Xi=0$, Eq. (27) reproduces the result of Ref. 63]: 


$$
\rho_{x x}^{(1 \mathrm{D})} \approx \frac{\pi^{2} b c T}{e^{2} v_{F}}\left|\tilde{\gamma}_{3}^{(1 \mathrm{D})}\left(\bar{q}_{x}, \bar{\varepsilon}\right)\right|^{2}
$$

The 1D resistance (29) is proportional to temperature $T$ multiplied by the square of the renormalized umklapp amplitude $\gamma_{3}$, which may also depend on temperature.

Now let us consider the simplest $2 \mathrm{D}$ case where only one tunneling amplitude $t_{b}$ is kept in Eq. (26). If $\varphi_{b}=0$ (similar equations hold also for $\varphi_{b}=\pi / 2$ ), then Eq. (26) can be factorized [52,53]:

$$
\left.\Xi\left(q_{y}, k_{y}, k_{y}^{\prime}\right)\right|_{\varphi_{b}=0}=8 t_{b} \cos \left(q_{y} b / 2\right) \cos \left[\left(k_{y}+k_{y}^{\prime}\right) b / 2\right] \cos \left[\left(k_{y}-k_{y}^{\prime}\right) b / 2\right] .
$$

If renormalization of $\gamma_{3}$ is neglected $\left(\gamma_{3}=g_{3}\right)$, then Eq. (27) with $\Xi$ from Eq. (30) gives:

$$
\left.\rho_{x x}^{(2 \mathrm{D})}\right|_{\varphi_{b}=0} \propto \tilde{g}_{3}^{2} T^{2} \ln ^{2}\left(t_{b} / T\right)
$$

which has an extra logarithmic factor compared to Eq. (28) 61]. Eqs. (28) and (31) are in agreement with the results of Ref. [29], where the so-called "hot spots" in the distribution of the umklapp scattering time over the Fermi surface of a Q1D metal were studied. The "hot spots" are the points where the scattering rate is strongly enhanced compared to the rest of the Fermi surface, typically by the factor $\ln \left(t_{b} / T\right)$ and occasionally by the factor $\sqrt[3]{t_{b} / T}$. Positions of the hot spots are determined by the saddle points of the function $\Xi\left(q_{y}, k_{y}, k_{y}^{\prime}\right)$ [29]. When $\varphi_{b} \neq 0, \pi / 2$, only isolated hot spots exist on the Fermi surface. Because they occupy a small phase space, the hot spots do not contribute significantly to resistivity in this general case, and Eq. (28) holds. However, in the special case $\varphi_{b}=0, \pi / 2$, the entire Fermi surface becomes "hot" [29], and resistivity acquires the logarithmic factor of Eq. (31).

In the special $2 \mathrm{D}$ case with only one tunneling amplitude $t_{b}$ and $\varphi_{b}=0$, the electron dispersion (11) has a perfect nesting at the wave vector $q_{x}=0$ and $q_{y}=\pi / b$. The zero-field susceptibility $\chi_{0}(\mathbf{q}, \varepsilon)$, given by Eqs. (12) and (6), diverges logarithmically at $\varepsilon=q_{x}=0$ and $q_{y}=\pi / b$. Since we neglect $g_{3}$ in the denominator of Eq. (10), the renormalized vertex $\gamma_{3}(0, \pi / b, 0)$ becomes

$$
\gamma_{3}(0, \pi / b, 0) \propto \frac{g_{3}}{g_{2}^{2} \ln ^{2}\left(T / T_{0}\right)}
$$


where $T_{0}=\hbar v_{F} /\left(\pi x_{0}\right) \exp \left(-1 / \tilde{g}_{2}\right)$ is the SDW transition temperature. At the same time, condition (25) with $\Xi$ given by Eq. (30) restricts integration in Eq. (27) to the vicinity of either $q_{y}=\pi / b$ or $k_{y}+k_{y}^{\prime}=\pi / b$ or $k_{y}-k_{y}^{\prime}=\pi / b$. The first of these conditions is satisfied at the same wave vector $q_{y}=\pi / b$ where $\gamma_{3}$ (32) diverges. Assuming that the integral in Eq. (27) is dominated by the vicinity of $q_{y}=\pi / b$, we find:

$$
\left.\rho_{x x}^{(2 \mathrm{D})}\right|_{\varphi_{b}=0} \propto \tilde{\gamma}_{3}^{2}(0, \pi / b, 0) T^{2} \ln ^{2}\left(t_{b} / T\right) \propto \frac{\tilde{g}_{3}^{2} T^{2} \ln ^{2}\left(t_{b} / T\right)}{\tilde{g}_{2}^{4} \ln ^{4}\left(T / T_{0}\right)}
$$

Eq. (33) is analogous to the expression obtained by Gor'kov [52,53], but differs in the powers of the logarithms. The factor $T^{2}$ in Eq. (33) tends to diminish resistivity with decreasing temperature, which is characteristic for a metal. On the other hand, the logarithmic factors in Eq. (33), both in the numerator and denominator, tend to increase resistance, which is characteristic for an insulator. Which of these two competing tendencies wins can be found numerically.

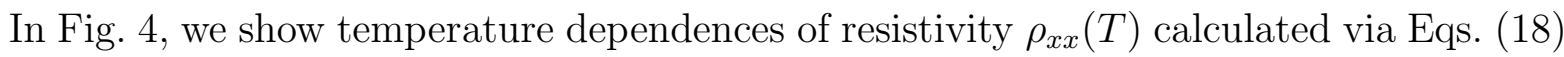
and (21) in 2D case at zero magnetic field. Because we neglected $g_{3}$ in the denominator of Eq. (10), $\rho_{x x} \propto g_{3}^{2}$ exactly. In Figs. 4, 5, and 6, we plot the ratio $\rho_{x x} / \tilde{g}_{3}^{2}$ where the dimensionless umklapp scattering amplitude $\tilde{g}_{3}=g_{3} / 2 \pi \hbar v_{F}$ cancels out. As discussed in Appendix, the value of $\tilde{g}_{3}$ can be recovered by comparing these figures with the experimental data. The top solid curve in Fig. 4 shows $\rho_{x x}(T)$ calculated with only one tunneling amplitude $t_{b}$ and $\varphi_{b}=0$. We observe that $\rho_{x x}(T)$ has a positive, metallic slope $d \rho_{x x}(T) / d T>0$ at high temperatures, whereas at lower temperatures the slope is negative. It is surprising that the negative slope starts at the temperature about $60 \mathrm{~K}$, which is several times higher than the SDW transition temperature $T_{0}=14.7 \mathrm{~K}$. This indicates that the logarithmic factors in Eq. (33) overcome the $T^{2}$ factor at relatively high temperatures.

Now let us discuss a more general 2D model with only one tunneling amplitude $t_{b}$, but with $\varphi_{b} \neq 0$. In this case, $\gamma_{3}$ diverges at the nesting vector $q_{x}=0$ and $q_{y}=(\pi-$ $\left.2 \varphi_{b}\right) / b$. The umklapp vertex $\gamma_{3}\left(0,\left(\pi-2 \varphi_{b}\right) / b, 0\right) \propto g_{3} / \ln \left(T / T_{0}\right)$ diverges at exactly the same transition temperature $T_{0}$ as in the case $\varphi_{b}=0$, but less strongly than in Eq. (32), 
because $\chi_{0}\left(0, q_{y}, 0\right)$ and $\chi_{0}\left(0,-q_{y}, 0\right)$ in Eq. (10) do not diverge at the same wave vector $q_{y}$. At $\varphi_{b} \neq 0$, condition (25) with $\Xi$ given by Eq. (26) is not satisfied at the nesting vector $q_{y}=\left(\pi-2 \varphi_{b}\right) / b$ independently of $k_{y}$ and $k_{y}^{\prime}$, which further reduces $\rho_{x x}(T)$ compared to Eq. (33) for $\varphi_{b}=0$. Temperature dependences $\rho_{x x}(T)$ are shown in Fig. 1 by solid curves for different values of $\varphi_{b}$. All curves have the same values of $t_{b}=290 \mathrm{~K}, \tilde{g}_{2}=0.2288$, and $T_{0}=14.7 \mathrm{~K}$ as the top solid curve with $\varphi_{b}=0$. While all solid curves diverge at the same transition temperature $T_{0}$, the region of the negative slope in $\rho_{x x}(T)$ shrinks rapidly with increasing $\varphi_{b}$ and becomes much smaller than $T_{0}$ at $\varphi_{b} \gtrsim \pi / 27$. Thus, a non-zero phase $\varphi_{b}$ strongly suppresses the precursor effect in resistivity. For the curves with $\varphi_{b} \gtrsim \pi / 27$, the behavior of $\rho_{x x}(T)$ qualitatively follows the 2D quadratic law (28) at the low temperatures $T \lesssim 2 t_{b} / \pi=185 \mathrm{~K}$ and the $1 \mathrm{D}$ law $(29)$ at the higher temperatures $T \gtrsim 2 t_{b} / \pi$. The slope of $\rho_{x x}(T)$ at $T \gtrsim 2 t_{b} / \pi$ is rather small, presumably because the $1 \mathrm{D}$ logarithmic renormalization of $\gamma_{3}$ partially compensates the linear temperature factor in Eq. (29).

The dotted curves in Fig. 1 represent $\rho_{x x}(T)$ for the 2D model where the tunneling amplitude to the next-nearest-neighboring chains, $t_{b}^{\prime}=20 \mathrm{~K}$, is introduced in addition to $t_{b}$. Since $t_{b}^{\prime}$ eliminates nesting in the dispersion law (1), the system does not have SDW instability provided $t_{b}^{\prime}>T_{0}$, so $\gamma_{3}$ does not diverge. The slope of the curve with $\varphi_{b}=0$ changes from negative to positive at $T \lesssim 2 t_{b}^{\prime} / \pi$, which creates a maximum in the $\rho_{x x}(T)$ curve. Thus, $t_{b}^{\prime}$ cuts off the precursor effect in $\rho_{x x}(T)$ at $T \sim t_{b}^{\prime} / \pi$, as discussed in Refs. $52,53$.

In conclusion, we have confirmed the suggestion by Gor'kov [52,53 that, in zero magnetic field, the renormalization of umklapp amplitude due to proximity to a SDW transition can produce a negative slope in $\rho_{x x}(T)$ at temperatures much higher than the SDW transition temperature $T_{0}$. This precursor effect is suppressed by a non-zero phase $\varphi_{b}$ in the electron dispersion law, which does not influence the SDW transition temperature, but shrinks the temperature region of the negative slope. The second tunneling amplitude $t_{b}^{\prime}$ suppresses the SDW transition temperature and cuts off the negative slope at $T \lesssim t_{b}^{\prime} / \pi$. In (TMTSF) ${ }_{2} \mathrm{X}$, negative slope of $\rho_{x x}(T)$ is not observed in zero magnetic field and appears only when 
a magnetic field is applied. According to the scenario suggested by Gor'kov 52,53, the negative slope is eliminated by a non-zero $t_{b}^{\prime}$ (and, possibly, by a non-zero $\varphi_{b}$, as shown above), but it is restored by a magnetic field. We examine feasibility of this scenario in the next section.

\section{TEMPERATURE DEPENDENCE OF RESISTIVITY IN A MAGNETIC FIELD}

In 2D case, the effect of a magnetic field on a Q1D electron system is characterized by the wave vector $G=e b H / \hbar c$ and the cyclotron energy $E_{H}=\hbar v_{F} G=e b H v_{F} / c$ 64. Using the parameters $v_{F}=2 \times 10^{5} \mathrm{~m} / \mathrm{s}$ and $b=7.7 \AA$, we find that $E_{H} / H \approx 1.8 \mathrm{~K} / \mathrm{T}$. Magnetic field enters Eqs. (18) and (19) for resistivity only through the function $\lambda\left(x, q_{y}\right)$ (6). In $2 \mathrm{D}$ case, magnetic field makes $\lambda$ a periodic function of $x$ with the period $2 \pi / G$ : $\lambda\left(x, q_{y}\right)=\lambda\left(x+2 \pi / G, q_{y}\right)$, so it can be expanded into a Fourier series:

$$
\lambda\left(x, q_{y}\right)=\sum_{n=-\infty}^{\infty} A_{n}\left(q_{y}\right) e^{i n G x}
$$

with some coefficients $A_{n}$. Substituting Eq. (34) into Eq. (19), we find:

$$
F(\mathbf{q}, \varepsilon)=\sum_{n=-\infty}^{\infty} \frac{A_{n}\left(q_{y}\right)}{\cosh \left(\frac{\varepsilon}{2 T}\right)+\cosh \left(\frac{\hbar v_{F}\left(q_{x}-n G\right)}{2 T}\right)}
$$

As follows from Eq. (35), the integration in Eq. (18) is concentrated in the energy interval $|\varepsilon| \lesssim T$ and, at $T \ll E_{H}$, in the vicinity of the integer wave vectors $q_{x} \approx n G$. Assuming that the characteristic width in $q_{x}$ and $\varepsilon$ of the function $\gamma_{3}(\mathbf{q}, \varepsilon)$ is greater than temperature, one could replace $\gamma_{3}(\mathbf{q}, \varepsilon)$ by $\gamma_{3}\left(n G, q_{y}, 0\right)$ in the integral (18). In this case, the integrals over $\varepsilon$ and $q_{x}$ can be taken analytically:

$$
\begin{aligned}
\rho_{x x}^{(2 D)} \approx & \frac{\pi^{2} b^{2} c T}{e^{2} v_{F}} \int d q_{y} \sum_{n=-\infty}^{\infty}\left|\tilde{\gamma}_{3}\left(n G, q_{y}, 0\right)\right|^{2} \\
& \times \sum_{n_{1}, n_{2}=-\infty}^{\infty} A_{n_{1}}\left(q_{y}\right) A_{n_{2}}\left(-q_{y}\right)\left(\frac{\left(n_{1}+n_{2}-n\right) E_{H} / 4 T}{\sinh \left[\left(n_{1}+n_{2}-n\right) E_{H} / 4 T\right]}\right)^{2} .
\end{aligned}
$$


At temperatures much lower than the cyclotron energy, $T \ll E_{H}$, only the term with $n_{1}+n_{2}=n$ contributes significantly to Eq. (36):

$$
\rho_{x x}^{(2 D)} \approx \frac{\pi^{2} b^{2} c T}{e^{2} v_{F}} \int d q_{y} \sum_{n=-\infty}^{\infty}\left|\tilde{\gamma}_{3}\left(n G, q_{y}, 0\right)\right|^{2} \sum_{n_{1}=-\infty}^{\infty} A_{n_{1}}\left(q_{y}\right) A_{n-n_{1}}\left(-q_{y}\right) .
$$

Eq. (37) is similar to the 1D formula (29), except for the additional integration over $q_{y}$ and summation over $n$. This is a consequence of one-dimensionalization of the electron spectrum (3) by the magnetic field.

If renormalization of $\gamma_{3}$ is neglected $\left(\gamma_{3}=g_{3}\right)$, then Eq. (37) produces a linear temperature dependence for resistivity in agreement with Lebed's results [20,45] for $T \ll E_{H}$. When renormalization of $\gamma_{3}$ is taken into account, $\gamma_{3}(\mathbf{q}, 0)$ diverges at a certain wave vector $q_{x}=N G$ and $q_{y}=Q_{y}$ as $T \rightarrow T_{c}$. Assuming that only the term with $q_{x}=N G$ dominates the sum (37), we find:

$$
\rho_{x x}^{(2 D)} \approx \frac{\pi^{2} b^{2} c T}{e^{2} v_{F}} \int d q_{y}\left|\tilde{\gamma}_{3}\left(N G, q_{y}, 0\right)\right|^{2} \sum_{n=-\infty}^{\infty} A_{n}\left(q_{y}\right) A_{N-n}\left(-q_{y}\right) .
$$

It is important to emphasize that the function $F(\mathbf{q}, \varepsilon)(35)$, while restricting integration over $\varepsilon$ and $q_{x}$, does not restricted integration over $q_{y}$ significantly (unlike in zero magnetic field, Sec. $\nabla I 1)$, because the Fourier coefficients $A_{n}\left(q_{y}\right)$ are nonsingular, temperature-independent functions of $q_{y}$. Thus, the integrals over $q_{y}$ in Eqs. (36)-(38) are not restricted to the vicinity of the FISDW vector $Q_{y}$. This does not allow us to replace $\gamma_{3}\left(N G, q_{y}, 0\right)$ by $\gamma_{3}\left(N G, Q_{y}, 0\right)$ and take the latter outside of the integral, like in Eq. (33). The integration over $q_{y}$ reduces the divergence of Eq. (38) at $T \rightarrow T_{c}$ and makes the resistivity precursor effect of SDW in a magnetic field weaker than without the field. This happens because the phase-space restrictions discussed in Sec. VII are consequences of the 2D nature of the electron dispersion law (1) at zero magnetic field, whereas a nonzero field makes the electron spectrum (3) onedimensional.

In Fig. 5, we show temperature dependences of resistivity calculated via Eqs. (18) and (35) for $t_{b}^{\prime}=20 \mathrm{~K}$, the phases $\varphi_{b}=0, \pi / 27$, and $\pi / 4$, and the magnetic fields $H=0,5,15$, and $25 \mathrm{~T}$. In the case of $\varphi_{b}=0$ (the top panel), only a very strong magnetic field $H=25$ 
T restores the negative slope of resistivity, $d \rho_{x x} / d T<0$. This result cannot be applied to explaining experiments [2 [4, because for $\varphi_{b}=0$ the slope of resistivity is already negative at $15<T<60 \mathrm{~K}$ in zero magnetic field, which does not agree with the experiment. On the other hand, once we increase $\varphi_{b}$ to make the zero-field slope positive, the effect of the magnetic field becomes very weak outside of a narrow vicinity of $T_{c}$ for both a very small

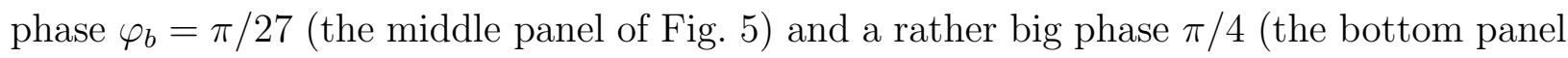
of Fig. 5). If we keep $\varphi_{b}=0$ and increase $t_{b}^{\prime}$ to the values $30 \mathrm{~K}$ or $40 \mathrm{~K}$, the zero-field slope becomes positive, but only an enormous magnetic field of $50 \mathrm{~T}$ makes the slope negative (see Fig. 6) [65]. One may conclude that in the case $\varphi_{b}=0$ the negative slope of resistance occupies a substantial range of magnetic fields only at very strong fields such that $E_{H}>t_{b}^{\prime}$ : $H=25 \mathrm{~T}$ for $t_{b}^{\prime}=20 \mathrm{~K}$ (the top panel of Fig. (5) and $H=50 \mathrm{~T}$ for $t_{b}^{\prime}=30$ and $40 \mathrm{~K}$ (Fig. (6). This scenario is hard to reconcile with the experiment, because, according to the standard theory of FISDW [54], the condition $E_{H}>t_{b}^{\prime}$ corresponds to the magnetic fields where the last, $N=0$ phase transition in the FISDW cascade takes place, whereas the negative slope in resistance occurs at much lower magnetic fields.

We conclude that the FISDW precursor scenario [52.53] cannot explain the anomalous temperature dependence of resistivity in Q1D conductors in a magnetic field observed in experiments [2 [- The insulating temperature dependence of resistivity, $d \rho_{x x} / d T<0$, does exist in this scenario at $T \gg T_{c}$ for a certain choice of the transverse dispersion law of electrons, however the effect is present even without magnetic field in this case. If the transverse dispersion law is modified to suppress the insulating behavior in zero field, then applying a magnetic field produces a negative slope in the temperature dependence of $\rho_{x x}(T)$ only either in a narrow vicinity of $T_{c}$ or at very strong fields such that $E_{H}>t_{b}^{\prime}$. 


\section{TEMPERATURE DEPENDENCE OF NUCLEAR MAGNETIC RELAXATION}

In Ref. [2], temperature dependence of the $\mathrm{NMR}$ relaxation rate in $(\mathrm{TMTSF})_{2} \mathrm{ClO}_{4}$ was measured and was discussed as an evidence for a charge pseudogap formation. In this section, we study the effect of a magnetic field on the NMR relaxation rate within the FISDW precursor scenario.

According to the theory of the NMR relaxation via the electron spin fluctuations 66 (see also Ref. [9]), the NMR relaxation rate $1 / T_{1}$ is proportional to the imaginary part of the electron spin susceptibility:

$$
\frac{1}{T_{1}} \propto T \sum_{\mathbf{q}} \frac{\operatorname{Im} \chi\left(\mathbf{q}, \omega_{n}\right)}{\omega_{n}}
$$

where $\omega_{n}$ is the nuclear Larmor frequency. The coefficient of proportionality in Eq. (39) depends on the hyperfine interaction parameters. In Q1D compounds, the sum over the wave vectors $\mathbf{q}$ in Eq. (39) can be separated into the contribution from the uniform spin susceptibility at $q_{x}$ close to $q_{x}=0$ and the contribution from the antiferromagnetic spin fluctuations at $q_{x}$ close to $q_{x}=2 k_{F}$ :

$$
T_{1}^{-1}=T_{1}^{-1}\left(q_{x} \approx 0\right)+T_{1}^{-1}\left(q_{x} \approx 2 k_{F}\right)
$$

In Refs. [2.67], the temperature dependences of each term in Eq. (40) were measured separately in (TMTSF) ${ }_{2} \mathrm{ClO}_{4}$ in the magnetic field $15 \mathrm{~T}$ along the $\mathbf{c}^{*}$ axis. It was found that the

antiferromagnetic term $T_{1}^{-1}\left(q_{x} \approx 2 k_{F}\right)$ starts to grow below the same temperature $T_{\min }$ that separates the metallic and insulating temperature dependences of resistivity $\left(d \rho_{x x} / d T>0\right.$ and $\left.d \rho_{x x} / d T<0\right)$. It was claimed that the growth of $T_{1}^{-1}\left(q_{x} \approx 2 k_{F}\right)$ is a manifestation of the opening of a charge pseudogap in the spectrum of electron excitations.

In the ladder approximation, the renormalized spin susceptibility $\chi(\mathbf{q}, \omega)$ is given by the Feynman diagrams similar to those shown in Figs. 1 and 2:

$$
\chi(\mathbf{q}, \omega)=\frac{\chi_{0}(\mathbf{q}, \omega)\left[1-g_{2} \chi_{0}(-\mathbf{q},-\omega)\right]}{\left[1-g_{2} \chi_{0}(\mathbf{q}, \omega)\right]\left[1-g_{2} \chi_{0}(-\mathbf{q},-\omega)\right]-g_{3}^{2} \chi_{0}(\mathbf{q}, \omega) \chi_{0}(-\mathbf{q},-\omega)} .
$$


Neglecting the umklapp splitting of the FISDW instability, i. e. setting $g_{3}=0$ in the denominator of (41), and taking the zero-frequency limit in Eq. (39), because the nuclear Larmor frequency is small compared to all other energies, we find:

$$
\frac{1}{T_{1}\left(q_{x} \approx 2 k_{F}\right)} \propto T \sum_{\mathbf{q}} \frac{\lim _{\omega \rightarrow 0} \operatorname{Im} \chi_{0}(\mathbf{q}, \omega) / \omega}{\left[1-g_{2} \chi_{0}(\mathbf{q}, 0)\right]^{2}} .
$$

$T_{1}^{-1}\left(q_{x} \approx 2 k_{F}\right)$ given by Eq. (42) does not depend on the phase $\varphi_{b}$. The temperature dependence of $T_{1}^{-1}\left(q_{x} \approx 2 k_{F}\right)$ calculated via Eq. (42) with $t_{b}^{\prime}=20 \mathrm{~K}$ is shown in Fig. 6. We see that $T_{1}^{-1}\left(q_{x} \approx 2 k_{F}\right)$ behaves in a magnetic field in the same way as resistivity does, i. e. it grows and deviates from the zero-field curve only in a narrow vicinity of the critical temperature. Thus, we conclude that the FISDW precursor scenario does not agree with the experimental behavior of the NMR relaxation rate in $(\mathrm{TMTSF})_{2} \mathrm{ClO}_{4}$ in a strong magnetic field. The results of our calculations are very similar to those of Ref. 68 for a SDW transition without magnetic field [55].

\section{CONCLUSIONS}

In this paper we presented a heuristic, semiphenomenological explanation of the anomalous temperature dependence of resistivity of Q1D conductors in a magnetic field observed in experiments 2 (4) reviewed in Sec. 国. According to this picture (Sec. II), a Q1D conductor behaves like an insulator $\left(d \rho_{x x} / d T<0\right)$, when its effective dimensionality is one, and like a metal $\left(d \rho_{x x} / d T>0\right)$, when its effective dimensionality is greater than one. Applying a magnetic field reduces the effective dimensionality of the system and switches the temperature dependence of resistivity between the insulating and metallic laws depending on the magnitude and orientation of the magnetic field. Using this picture, we predicted that the Danner oscillations of $\rho_{c c}$ may be observed when a magnetic field is rotated in the magic plane from the $\mathbf{b}+\mathbf{c}$ direction toward the a direction. We critically analyzed whether various microscopic models suggested in literature can justify our heuristic picture and found that none of the models is fully satisfactory (Sec. [II). We studied the FISDW precursor scenario 
suggested by Gor'kov [52,53 in detail both analytically and numerically (Sec. [V] [X]) and found that it does not agree with the experimental observations. In the rest of this section, we speculate about possible alternative approaches to solving the problem.

1. Within the FISDW precursor scenario, we studied only the umklapp scattering rate $1 / \tau$. In the language of Feynman diagrams, $1 / \tau$ is related to the imaginary part of the electron self-energy $\Sigma$. Via the Kramers-Kronig relations for $\Sigma$, any precursor effects in $\operatorname{Im} \Sigma$ should also affect $\operatorname{Re} \Sigma$. Because $\operatorname{Re} \Sigma$ is related to the electron density of states, a pseudogap may open in the single-electron spectral density as a precursor of FISDW. Potentially, the pseudogap may affect transport properties of the system as strongly as the renormalization of scattering rate studied in this paper. $\operatorname{Re} \Sigma$ also determines the residue $Z$ of the single-electron Green function, which is finite for a Fermi liquid and vanishes for a Luttinger liquid. $\operatorname{Re} \Sigma$ can be straightforwardly calculated using the methods of this paper. On the other hand, relationship between the single-electron and transport properties is not straightforward. For example, the residue $Z$ may cancel out from resistivity due to the Ward identities [69]. Reliable calculations of resistivity require taking into account corrections to the vertex of interaction between electrons and electromagnetic field, which is a difficult problem for inelastic electron-electron interaction [70].

2. The ladder approximation utilized in this paper does not take into account contributions from superconducting channel, which, on one hand, plays a very important role in one dimension and, on the other hand, is sensitive to a magnetic field. The magnetic field may induce an insulating behavior by suppressing superconducting fluctuations, which compensate insulating fluctuations at zero magnetic field. Including both density-wave and superconducting channels naturally leads to the parquet equations. If the parquet equations in a magnetic field [31,49,50] are generalized to include the umklapp amplitude, one may try to find out whether they can be decomposed into two disconnected sets of equation analogously to the spin-charge separation in 1D case. This is a nontrivial possibility, because the number of coupled equations is infinite, and the equations are nonlinear. Nevertheless, if the equations do decouple, the two sets of equations would diverge at two different tem- 
peratures, one of which could be identified with $T_{\min }$ and another with $T_{c}$. An additional technical problem is that the equations of Refs. 31,49,50 are applicable only at $T \lesssim E_{H}$, whereas $T_{\min } \sim E_{H}$, so the parquet equations need to be derived in the difficult range

$T \gtrsim E_{H}$. The parquet approach may require to assume a rather small effective value for $t_{b}$ [71,72.

3. Electron-electron scattering contributes to electrical resistivity only via the umklapp processes, which do not conserve the total electron momentum [60]. For commensurate systems, such as (TMTSF $)_{2} \mathrm{X}$, the umklapp scattering process that changes the total electron momentum by $4 k_{F}=2 \pi / a$, where $a$ is the lattice spacing in the a direction, is usually considered. However, in the presence of a magnetic field, the total electron momentum may also change by a multiple of $G$, the wave vector of the magnetic field. Since this kind of umklapp exists only in a magnetic field, it may be a natural source of magnetoresistance in a Q1D system. It is reasonable to expect that this mechanism works effectively only at $T \lesssim E_{H}$. This could generate $T_{\min } \sim E_{H}$ without invoking pseudogaps originating from renormalization, whose values tend to be close to $T_{c}$. On the other hand, this mechanism would not explain the insulating behavior of the transverse resistivities $\rho_{y y}$ and $\rho_{z z}$ and the NMR relaxation rate. However, the recent experiment [24], where metallic temperature dependence was found for $\rho_{z z}$ and insulating for $\rho_{x x}$ and $\rho_{y y}$, may be in favor of this or another kinetic mechanism and against a charge-gap scenario.

Undoubtedly, the anomalous temperature dependence of resistivity in Q1D conductors in a magnetic field poses a tough theoretical challenge and solving this difficult puzzle would greatly enrich the condensed-matter physics.

\section{ACKNOWLEDGMENTS}

We are grateful to P. M. Chaikin, N. Dupuis, L. P. Gor'kov, and D. Jérome for useful discussions. We also thank L. Balicas, E. Chashechkina, and J. Moser for sharing their unpublished experimental data with us. This work was partially supported by the NSF 
under grant DMR-9417451 and by the David and Lucile Packard Foundation.

\section{APPENDIX}

The absolute values of $\rho_{x x}$ in (TMTSF $)_{2} \mathrm{PF}_{6}$ can be found in Figs. 1 and 2 of Ref. [6], Fig. 20 of Ref. [9], Fig. 4 of Ref. [13], and Figs. 2 and 3 of Ref. [61]. From these data, as well as from Ref. [73], we find that $\rho_{x x} \approx 18 \mu \Omega \mathrm{cm}$ at $T=20 \mathrm{~K}$. Comparing this experimental value of resistivity with the theoretical curves in Fig. 4, we find $\tilde{g}_{3}=0.17$ at $\varphi_{b}=0$ or $\tilde{g}_{3}=0.77$ at $\varphi_{b}=\pi / 4$. On the other hand, taking the value $\rho_{x x} \approx 1 \mathrm{~m} \Omega \mathrm{cm}$ at $T=300 \mathrm{~K}$ [6,9], we would find $\tilde{g}_{3} \approx 1$.

In Ref. [61], the experimental temperature dependence of $\rho_{x x}$ in $(\mathrm{TMTSF})_{2} \mathrm{PF}_{6}$ was fit

neglecting renormalization of $\gamma_{3}$, and the value $g_{3}^{(G M)}=0.21$ was found. However, because our Eq. (13) differs from Eq. (3) of Ref. 61 by a factor $(2 \pi)^{3}$, and a factor 0.5 is missing in Eq. (7) of Ref. [61], the value $g_{3}^{(G M)}=0.21$ corresponds to $\tilde{g}_{3}=2 \pi^{3 / 2} g_{3}^{(G M)}=2.34$ in our notation. The ratio of this value and the values quoted in the preceding paragraph, $2.34 / 0.77=3.3$, represents the effect of renormalization of $\gamma_{3}$ by the SDW diagrams.

According to the Drude formula, resistivity along the chains is

$$
\rho_{x x}=\frac{\pi b c \hbar}{2 e^{2} v_{F} \tau}=\frac{4 \pi}{\omega_{p}^{2} \tau},
$$

where $\tau$ is the relaxation rate, and $\omega_{p}^{2}=8 e^{2} v_{F} / b c \hbar$ is the plasma frequency. Using the values of $v_{F}, b$ and $c$, we find $\omega_{p}=970 \mathrm{~cm}^{-1}$, which is close to the value $\omega_{p}=1.1 \times 10^{4} \mathrm{~cm}^{-1}$ from Ref. [61]. Comparing Eq. (43) with the quoted above experimental values of $\rho_{x x}$, we find $1 / \tau \approx 40 \mathrm{~K}$ at $T=20 \mathrm{~K}$ and $1 / \tau \approx 2200 \mathrm{~K}$ at $T=300 \mathrm{~K}$. One may notice that both of these values for $1 / \tau$ are greater than the corresponding temperatures: $1 / \tau>T$. For that reason, it may be more appropriate to use $1 / \tau$, rather than $T$, as an infrared cutoff of the renormalization. 


\section{REFERENCES}

* Now at MetricVision, a Thermo Electron Company.

$\dagger \quad$ E-mail yakovenk@physics.umd.edu.

[1] J. R. Cooper and B. Korin-Hamzić in Organic Conductors: Fundamentals and Applications, edited by J.-P. Farges (Marcel Dekker, New York, 1994), p. 389.

[2] K. Behnia, L. Balicas, W. Kang, D. Jérome, P. Carretta, Y. Fagot-Revurat, C. Berthier, M. Horvatić, P. Ségransan, L. Hubert, and C. Bourbonnais, Phys. Rev. Lett. 74, 5272 (1995).

[3] D. Jérome, in Correlated Fermions and Transport in Mesoscopic Systems, edited by T. Martin, G. Montambaux, and J. Trân Thanh Vân (Editions Frontieres, Gif-sur-Yvette, 1996), p. 95.

[4] E. I. Chashechkina and P. M. Chaikin, Phys. Rev. Lett. 80, 2181 (1998).

[5] TMTSF is tetramethyltetraselenafulvalene.

[6] C. S. Jacobsen, K. Mortensen, M. Weger, and K. Bechgaard, Sol. State Comm. 38, 423 (1981).

[7] L. Forró, K. Biljaković, J. R. Cooper, and K. Bechgaard, Phys. Rev. B 29, 2839 (1984).

[8] J. R. Cooper, L. Forró, B. Korin-Hamzić, K. Bechgaard, and A. Moradpour, Phys. Rev. B 33, 6810 (1986).

[9] D. Jérome, in Organic Conductors: Fundamentals and Applications, edited by J.-P. Farges (Marcel Dekker, New York, 1994), p. 405.

[10] T. Ishiguro and K. Yamaji, Organic Superconductors (Springer-Verlag, Berlin, 1990).

[11] L. P. Gor'kov, Usp. Fiz. Nauk 144, 381 (1984) [Sov. Phys. Usp. 27, 809 (1984)].

[12] D. Jérome and H. J. Schulz, Adv. Phys. 31, 299 (1982). 
[13] J. Moser, M. Gabay, P. Auban-Senzier, D. Jérome, K. Bechgaard, and J. M. Fabre, Eur. Phys. J. B 1, 39 (1998).

[14] A. A. Abrikosov, L. P. Gorkov, and I. E. Dzyaloshinskii, Methods of Quantum Field Theory in Statistical Physics (Dover, New York, 1975).

[15] In Refs. [2, [3], temperature dependence of resistance was measured for a single orientation of the magnetic field parallel to the $\mathbf{c}^{*}$ axis.

[16] T. Osada, A. Kawasumi, S. Kagoshima, N. Miura, and G. Saito, Phys. Rev. Lett. 66, $1525(1991)$.

[17] M. J. Naughton, O. H. Chung, M. Chaparala, X. Bu, and P. Coppens, Phys. Rev. Lett. 67, 3712 (1991).

[18] W. Kang, S. T. Hannahs, and P. M. Chaikin, Phys. Rev. Lett. 69, 2827 (1992).

[19] A. G. Lebed, Pis'ma v Zh. Eksp. Teor. Fiz. 43, 137 (1986) [JETP Lett. 43, 174 (1986)].

[20] A. G. Lebed' and P. Bak, Phys. Rev. Lett. 63, 1315 (1989).

[21] G. Danner, W. Kang, and P. M. Chaikin, Physica B 201, 442 (1994).

[22] L. Balicas, K. Behnia, W. Kang, D. Jérome, L. Hubert, and C. Bourbonnais, Phys. Rev. Lett. 78, 984 (1997).

[23] L. Balicas, preprint.

[24] E. I. Chashechkina and P. M. Chaikin, to be published in Synth. Metals (Proceedings of ICSM'98).

[25] J. Moser (private communication).

[26] The results shown in Fig. 1 of Ref. [2] are somewhat spoiled by the fact that the two sets of measurements were performed in two different laboratories, which resulted in different cooling rates of the sample and a big gap between the curves corresponding to 
the very close fields $H=12.3 \mathrm{~T}$ and $12.5 \mathrm{~T}$.

[27] G. M. Danner, N. P. Ong, and P. M. Chaikin, Phys. Rev. Lett. 78, 983 (1997).

[28] E. I. Chashechkina and P. M. Chaikin, Phys. Rev. B 56, 13658 (1997).

[29] A. T. Zheleznyak and V. M. Yakovenko, Synth. Metals 70, 1005 (1995).

[30] This heuristic picture was presented by V. M. Yakovenko in conference talks at Workshop on Non-Fermi Liquid in One Dimension (UCLA, 1995), Correlated Fermions and Transport in Mesoscopic Systems (Les Arcs, 1996), Non-Fermi-Liquid Physics (ITP, Santa Barbara, 1996) (unpublished), and International Conference on Synthetic Metals (Montpellier, 1998) (to be published in Synth. Metals, cond-mat/9807061).

[31] V. M. Yakovenko, Zh. Eksp. Teor. Fiz. 93, 627 (1987) [Sov. Phys. JETP 66, 355 (1987)].

[32] At $T<T_{\max }, \rho_{a a}$ indeed depends only on the projection of the magnetic field on the $\mathbf{c}^{*}$ axis for the nonmagic orientations of the magnetic field [39,44]. At $T>T_{\max }$, the projection dependence should hold, presumably, for all orientations, because the magic angles effect does not exist at $T>T_{\max }$.

[33] P. M. Grant, J. Phys. (France), Colloque C3, 44, Suppl. 6, C3-847 (1983).

[34] G. M. Danner and P. M. Chaikin, Phys. Rev. Lett. 75, 4690 (1995).

[35] C. S. Jacobsen, D. B. Tanner, and K. Bechgaard, Phys. Rev. B 28, 7019 (1983).

[36] L. P. Gor'kov and A. G. Lebed', J. Phys. Lett. (France) 45, L433 (1984).

[37] F. D. M. Haldane, J. Phys. C 14, 2585 (1981).

[38] G. M. Danner, W. Kang, and P. M. Chaikin, Phys. Rev. Lett. 72, 3714 (1994).

[39] S. P. Strong, D. G. Clarke, and P. W. Anderson, Phys. Rev. Lett. 73, 1007 (1994).

[40] I. J. Lee and M. J. Naughton, Phys. Rev. B 57, 7423 (1998); to be published in Synth. Metals (Proceedings of ICSM'98). 
[41] K. Maki, Phys. Rev. B 45, 5111 (1992).

[42] T. Osada, S. Kagoshima, and N. Miura, Phys. Rev. B 46, 1812 (1992).

[43] P. M. Chaikin, Phys. Rev. Lett. 69, 2831 (1992).

[44] D. G. Clarke, S. P. Strong, P. M. Chaikin, and E. I. Chashechkina, Science 279, 2071 (1998).

[45] A. G. Lebed, J. Phys. I (France) 4, 351 (1994); Synth. Metals 70, 993 (1995); J. Phys. I (France) 6, 1819 (1996).

[46] C. Coulon, F. Delhaes, S. Flandrois, R. Lagnier, E. Bonjour, and J. M. Fabre, J. Phys. (France) 43, 1059 (1982).

[47] I. E. Dzyaloshinskii and A. I. Larkin, Zh. Exp. Teor. Fiz. 61, 791 (1972) [Sov. Phys. JETP 34, 422 (1972)].

[48] L. P. Gor'kov and I. E. Dzyaloshinskii, Zh. Exp. Teor. Fiz. 67, 397 (1974) [Sov. Phys. JETP 40, 198 (1975)].

[49] V. M. Yakovenko, Europhys. Lett. 31041 (1987).

[50] V. M. Yakovenko, Phys. Rev. B 43, 11353 (1991).

[51] L. D. Landau and E. M. Lifshitz, Statistical Physics, Part 1 (Pergamon, Oxford, 1993), p. 537 .

[52] L. P. Gor'kov, Europhys. Lett. 31, 49 (1995).

[53] L. P. Gor'kov, J. Phys. I (France) 6, 1697 (1996).

[54] G. Montambaux, M. Héritier, and P. Lederer, Phys. Rev. Lett. 55, 2078 (1985).

[55] Technically, the high-energy cutoff $\hbar v_{F} / \pi x_{0} \sim \varepsilon_{F} \sim t_{a}$ that we use for the calculation $\chi_{0}$ is not quite appropriate, because at the energies $\varepsilon \geq t_{b}$ the system is truly onedimensional, and the Peierls instability is cancelled by the Cooper one in the parquet 
approximation [31]. So the more appropriate energy cutoff would be $t_{b}$, which is much smaller than $\hbar v_{F} / \pi x_{0}$. Nevertheless, it appears that changing the cutoff would only affect the numbers and would not alter the qualitative conclusions of the paper.

[56] K. Yamaji, J. Phys. Soc. Jpn. 55, 860 (1986).

[57] A. G. Lebed', Physica Scripta T 39, 386 (1991); Zh. Eksp. Teor. Fiz. 99, 1849 (1991) [Sov. Phys. JETP 72, 1035 (1991)].

[58] For a systematic study of the effect of umklapp on the FISDW state, see N. Dupuis and V. M. Yakovenko, Phys. Rev. Lett. 80, 3618 (1998); Phys. Rev. B 58, 8773 (1998).

[59] P. M. Chaikin, W. Kang, S. T. Hannahs, and R. C. Yu, Physica B 177, 353 (1992).

[60] J. M. Ziman, Electrons and Phonons (Oxford University Press, Oxford, 1960).

[61] L. P. Gor'kov and M. Mochena, Phys. Rev. B 57, 6204 (1998).

[62] By $1 \mathrm{D}$ or $2 \mathrm{D}$ case we mean that $t_{b}=0$ or $t_{c}=0$; that is, either the chains or the plains of a Q1D conductor are decoupled. However, in all cases, the expressions for $\rho_{x x}$ in this paper have the dimensionality of 3D resistivity. Resistivity of a single chain would be given by Eq. (29) divided by $b c$.

[63] L. P. Gor'kov and I. E. Dzyaloshinskii, Pis'ma Zh. Eksp. Teor. Fiz. 18, 686 (1973) [JETP Lett. 18, 401 (1973)].

[64] More precisely, the expressions for $G$ and $E_{H}$ should contain not the lattice spacing $b$, but its projection perpendicular to the a axis; however, the difference is not significant.

[65] Increasing $t_{b}^{\prime}$ to $30 \mathrm{~K}$ or $40 \mathrm{~K}$ also changes the phase diagram of the system shown in Fig. 辽.

[66] T. Moriya, J. Phys. Soc. Jpn., 18, 516 (1963).

[67] P. Carretta, C. Berthier, M. Horvatić, Y. Fagot-Revurat, P.Ségransan, D. Jérome, and 
C. Bourbonnais, in Physical Phenomena in High Magnetic Fields - II, edited by Z. Fisk, L. Gor'kov, D. Meltzer, and R. Schrieffer (World Scientific, Singapore, 1996), p. 328.

[68] C. Bourbonnais, J. Phys. I (France) 3, 143 (1993).

[69] P. Nozières, Theory of Interacting Fermi Systems (W. A. Benjamin New York, 1964).

[70] G. D. Mahan, Many-Particle Physics, 2nd ed. (Plenum Press, New York, 1990).

[71] S. A. Brazovskii and V. M. Yakovenko, Zh. Eksp. Teor. Fiz. 89, 2318 (1985) [Sov. Phys. JETP 62, 1340 (1985)].

[72] V. M. Yakovenko, Phys. Rev. Lett. 61, 2276 (1988).

[73] D. Jérome (private communication). 


\section{FIGURES}

FIG. 1. Feynman diagram for the bare density-wave susceptibility $\chi_{0}\left(x^{\prime}-x, \mathbf{q}_{\perp}, i \Omega_{m}\right)$. The solid and dashed lines represent the Green functions of the + and - electrons.

FIG. 2. Feynman diagrams for the renormalized vertices of forward $\left(\gamma_{2}\right)$ and umklapp $\left(\gamma_{3}\right)$ scattering in the ladder approximation. The wavy lines represent the bare, unrenormalized vertices of forward $\left(g_{2}\right)$ and umklapp $\left(g_{3}\right)$ scattering, whereas the circles represent the corresponding renormalized vertices. The + and - electron lines (the solid and dashed lines) are implied to have opposite spins. Spin is conserved along the thin lines inside the circles.

FIG. 3. Phase diagram of a Q1D conductor in the temperature (T) vs magnetic field (H) plane

calculated from Eq. (11) with neglected umklapp interaction $g_{3}=0$. Various symbols denote the integer values $N$ of the quantized wave vector of FISDW, $q_{x}=N G$.

FIG. 4. Temperature dependences of longitudinal resistivity normalized to the dimensionless umklapp scattering amplitude, $\rho_{x x}(T) / \tilde{g}_{3}^{2}$, at zero magnetic field for different values of the phase $\varphi_{b}$. The solid lines correspond to $t_{b}^{\prime}=0$, in which case resistivity diverges at $T \rightarrow T_{0}$, where $T_{0}=14.7 \mathrm{~K}$ is the SDW transition temperature. The dotted lines correspond to $t_{b}^{\prime}=20 \mathrm{~K}$, in which case there is no SDW transition at zero magnetic field.

FIG. 5. Temperature dependences of the umklapp resistivity at $t_{b}^{\prime}=20 \mathrm{~K}$ for different values of the phase $\varphi_{b}$ in the magnetic fields 5,15 , and $25 \mathrm{~T}$ (curves a, b, and c, respectively) and without magnetic field (dots).

FIG. 6. Temperature dependences of the umklapp resistivity at $t_{b}^{\prime}=30 \mathrm{~K}$ (top panel) and $t_{b}^{\prime}=40 \mathrm{~K}$ (bottom panel) for $\varphi_{b}=0$ in the magnetic fields 5, 25, and $50 \mathrm{~T}$ (curves a, b, and c, respectively) and without magnetic field (dots).

FIG. 7. Temperature dependences of the $2 k_{F}$ component of the NMR relaxation rate (in arbitrary units) at zero (dots) and nonzero (solid curves) magnetic field for $t_{b}^{\prime}=20 \mathrm{~K}$. 


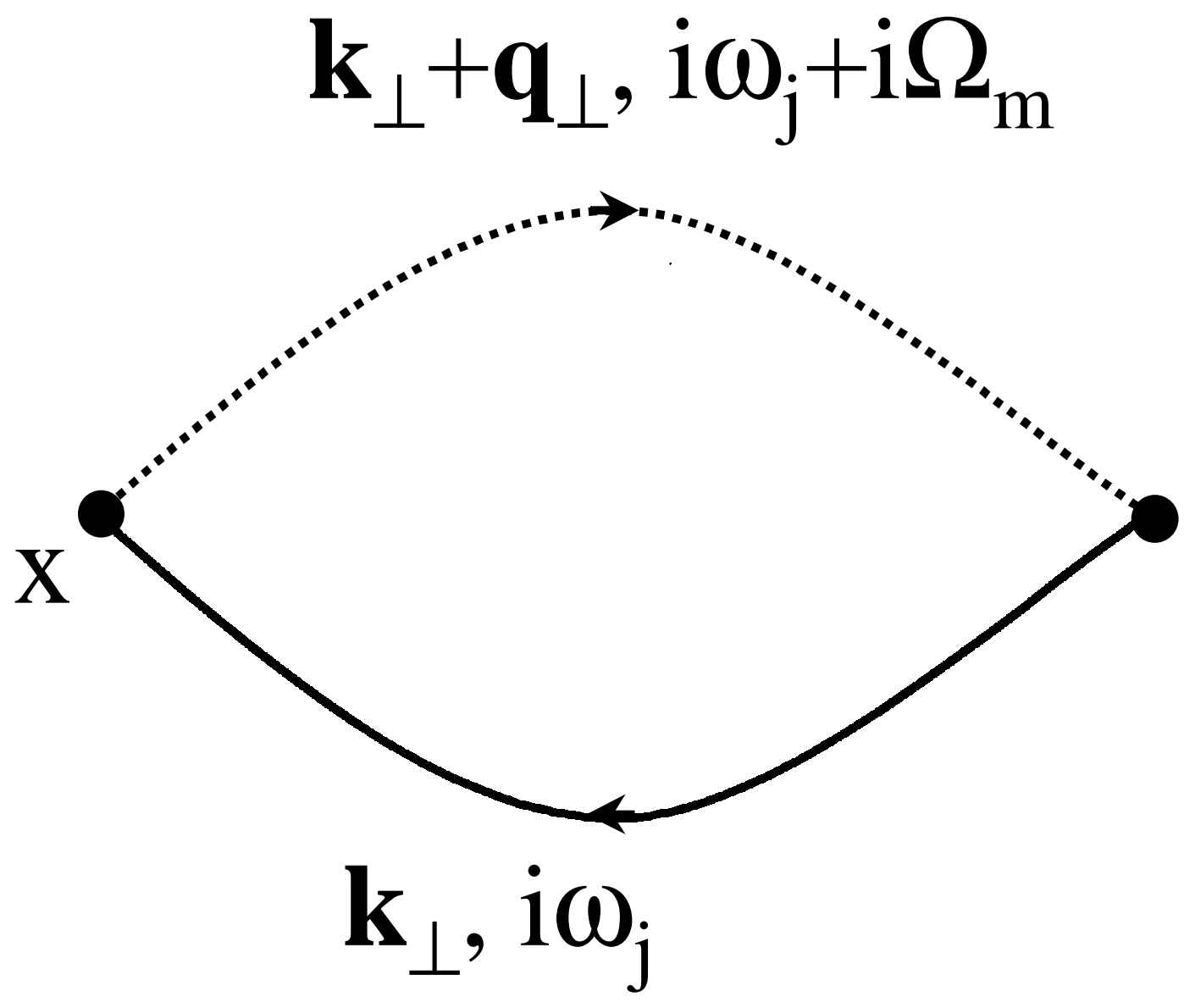




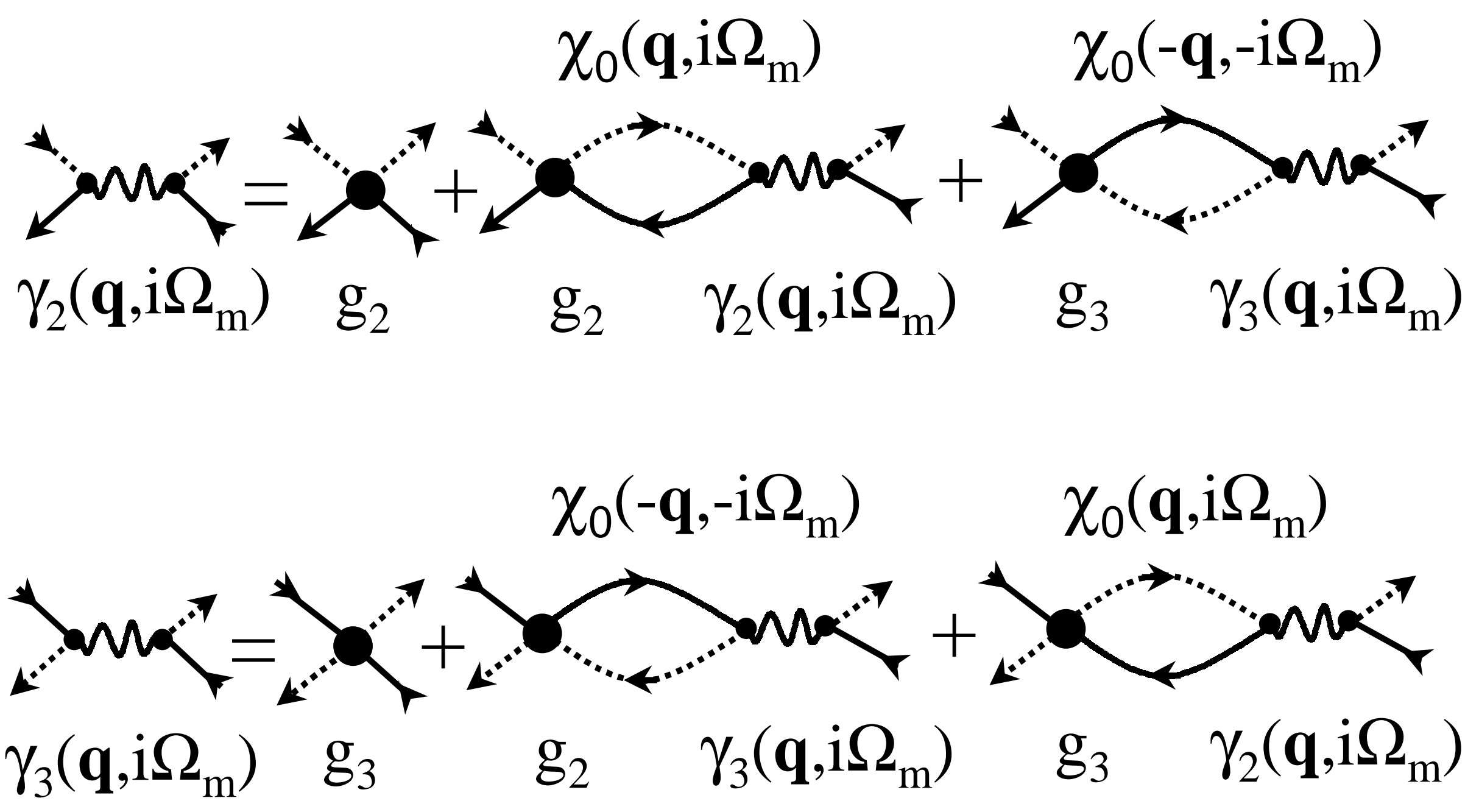




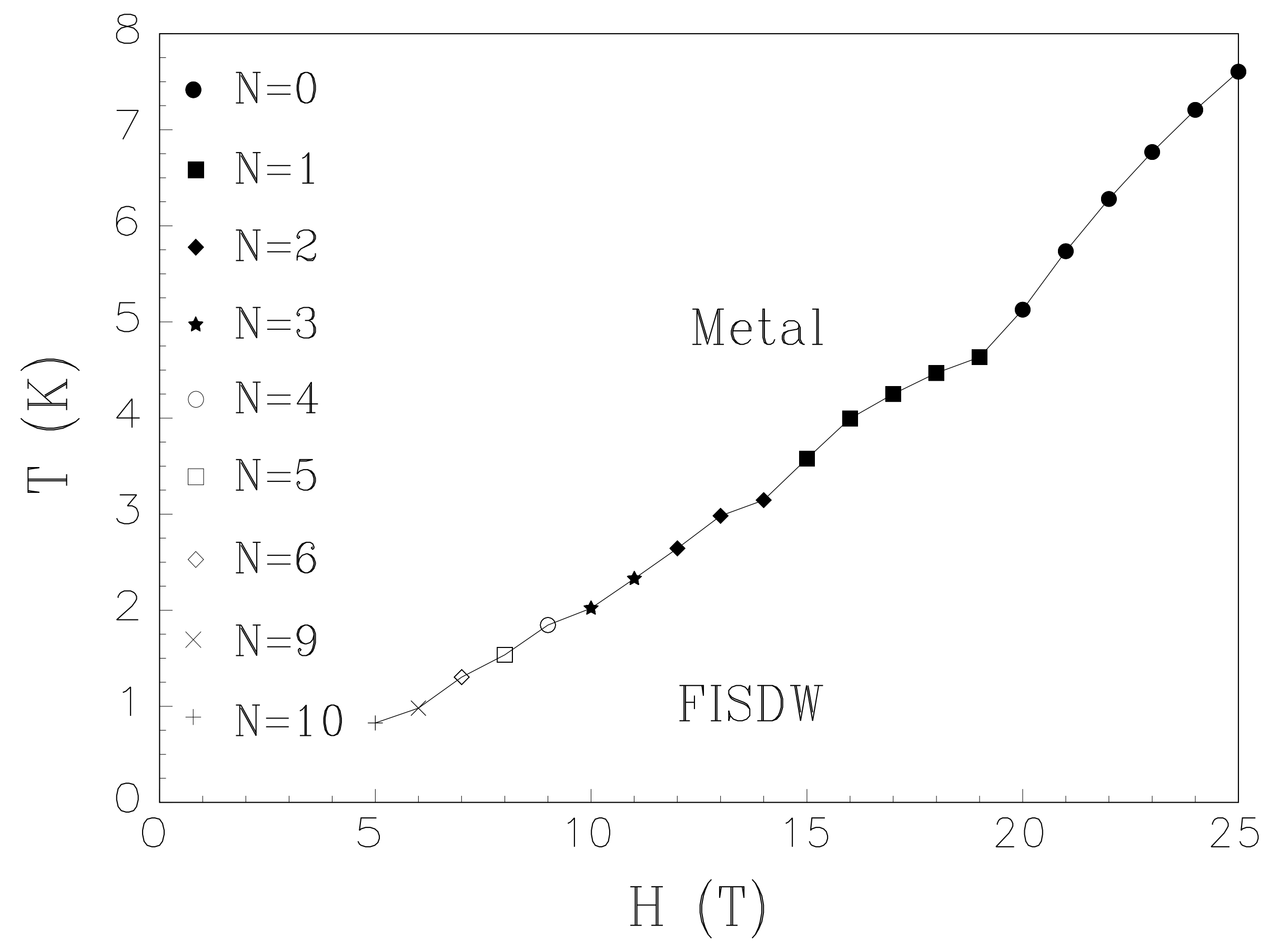




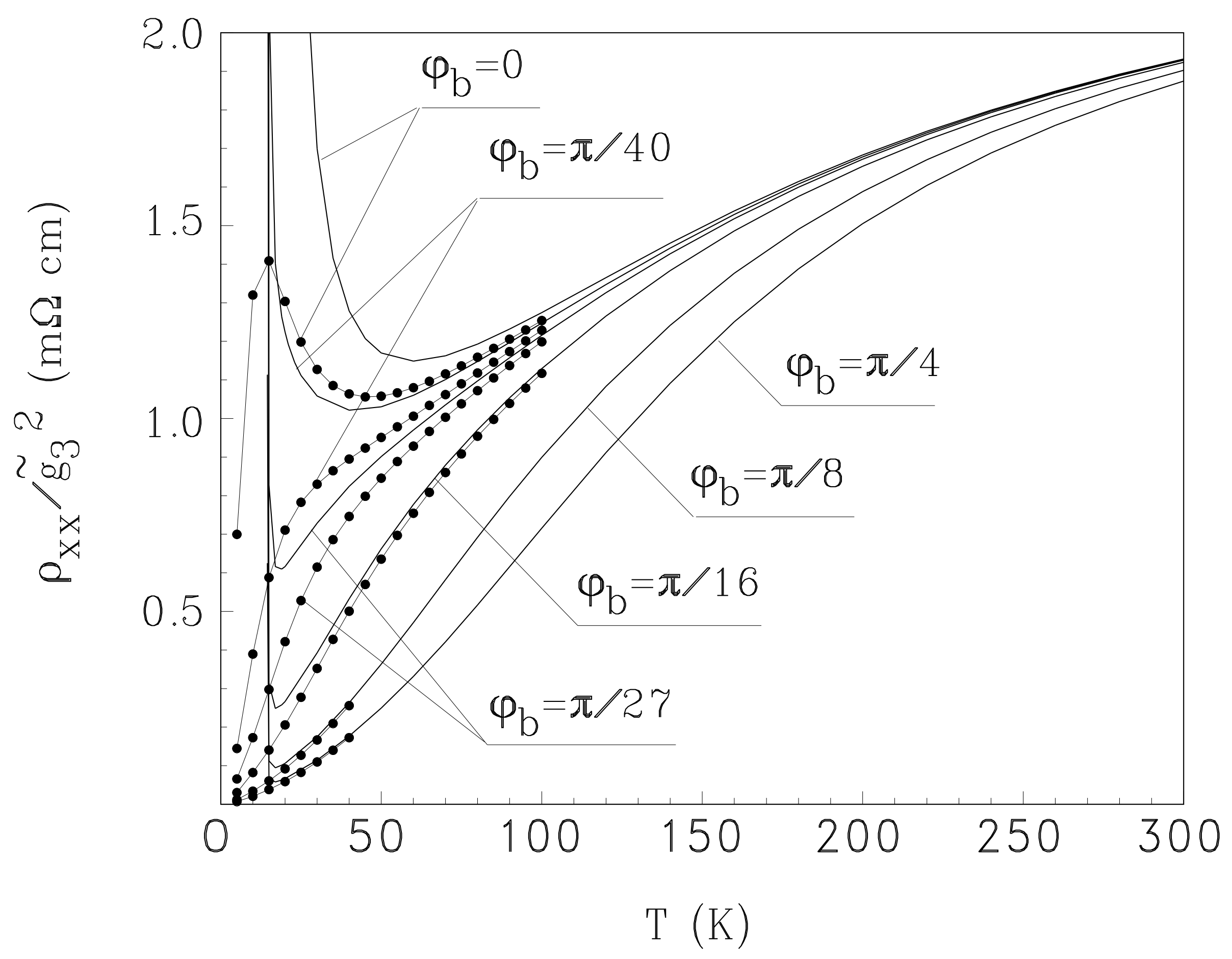




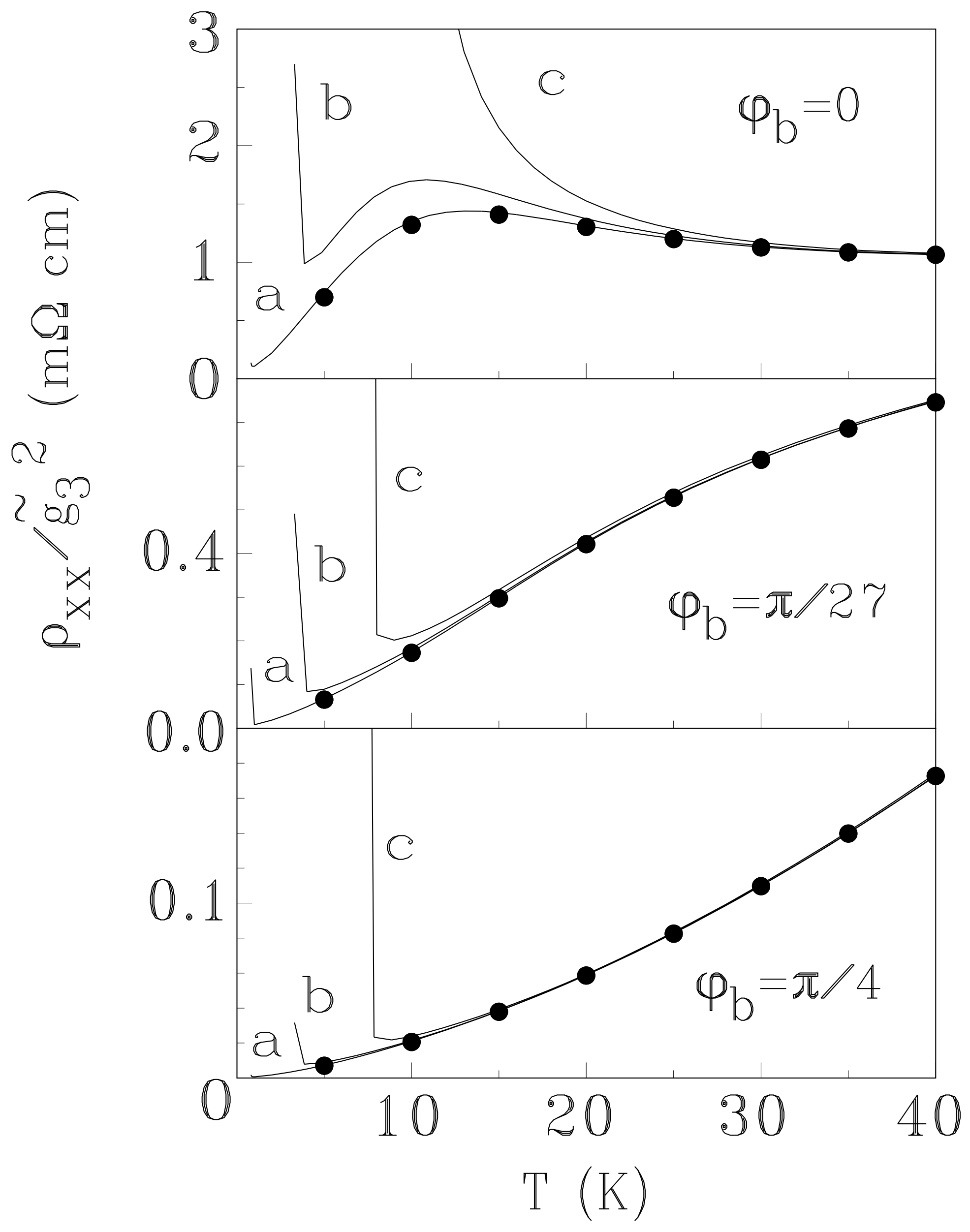




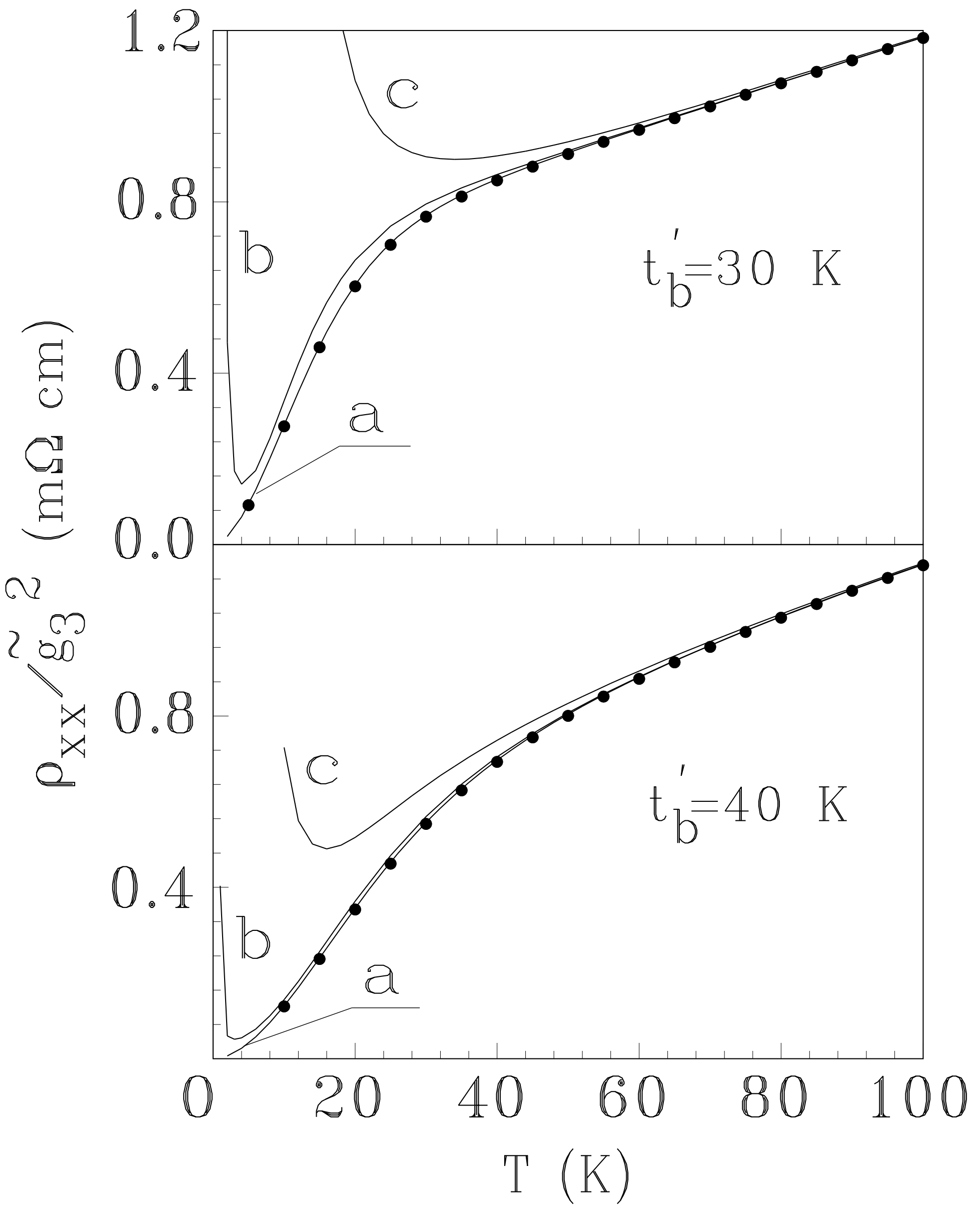




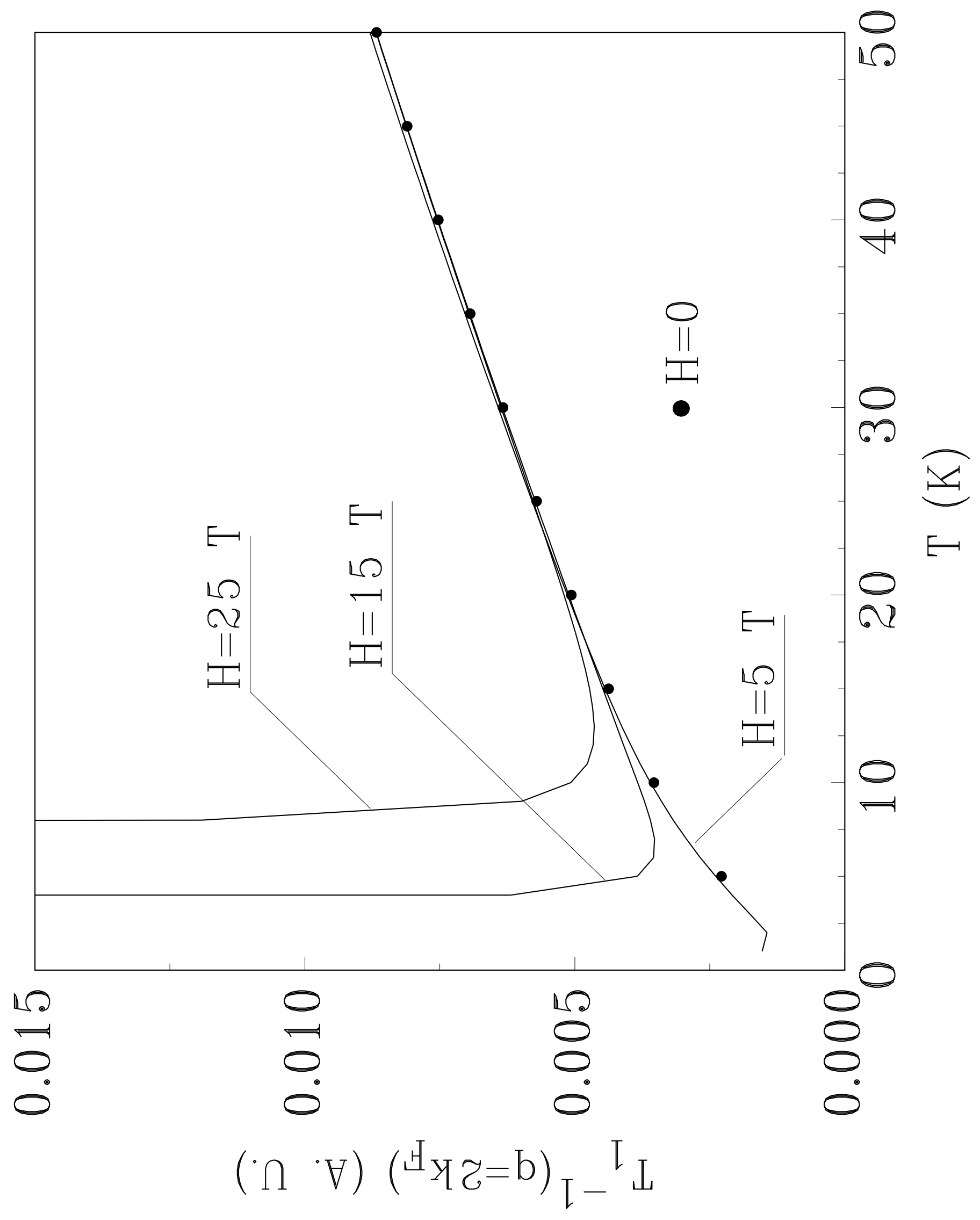

